\title{
Quantitative Reasoning for Proving Lock-Freedom
}

\author{
Jan Hoffmann \\ Yale University
}

\author{
Michael Marmar \\ Yale University
}

\author{
Zhong Shao \\ Yale University
}

\begin{abstract}
This article describes a novel quantitative proof technique for the modular and local verification of lock-freedom. In contrast to proofs based on temporal rely-guarantee requirements, this new quantitative reasoning method can be directly integrated in modern program logics that are designed for the verification of safety properties. Using a single formalism for verifying memory safety and lock-freedom allows a combined correctness proof that verifies both properties simultaneously.

This article presents one possible formalization of this quantitative proof technique by developing a variant of concurrent separation logic (CSL) for total correctness. To enable quantitative reasoning, CSL is extended with a predicate for affine tokens to account for, and provide an upper bound on the number of loop iterations in a program. Lock-freedom is then reduced to total-correctness proofs. Quantitative reasoning is demonstrated in detail, both informally and formally, by verifying the lockfreedom of Treiber's non-blocking stack. Furthermore, it is shown how the technique is used to verify the lock-freedom of more advanced shared-memory data structures that use eliminationbackoff schemes and hazard-pointers.
\end{abstract}

\section{INTRODUCTION}

The efficient use of multicore and multiprocessor systems requires high performance shared-memory data structures. Performance issues with traditional lock-based synchronization has generated increasing interest in non-blocking sharedmemory data structures. In many scenarios, non-blocking data structures outperform their lock-based counterparts [1], [2]. However, their optimistic approach to concurrency complicates reasoning about their correctness.

A non-blocking data structure should guarantee that any sequence of concurrent operations that modify or access the data structure do so in a consistent way. Such a guarantee is a safety property which is implied by linearizability [3]. Additionally, a non-blocking data structure should guarantee certain liveness properties, which ensure that desired events eventually occur when the program is executed, independent of thread contention or the whims of the scheduler. These properties are ensured by progress conditions such as obstruction-freedom, lock-freedom, and wait-freedom [4], [5] (see §II). In general, it is easier to implement the data structure efficiently if the progress guarantees it makes are weaker. Lock-freedom has proven to be a sweet spot that provides a strong progress guarantee and allows for elegant and efficient implementations in practice [6], [7], [8], [9].

The formal verification of practical lock-free data structures is an interesting problem because of their relevance and the challenges they bear for current verification techniques: They employ fine-grained concurrency, shared-memory pointer-based data structures, pointer manipulation, and control flow that depends on shared state.

Classically, verification of lock-freedom is reduced to modelchecking liveness properties on whole-program execution traces [10], [11], [12]. Recently, Gotsman et al. [13] have argued that lock-freedom can be reduced to modular, threadlocal termination proofs of concurrent programs in which each thread only executes a single data-structure operation. Termination is then proven using a combination of concurrent separation logic (CSL) [14] and temporal trace-based relyguarantee reasoning. In this way, proving lock-freedom is reduced to a finite number of termination proofs which can be automatically found. However, as we show in $\S \mathrm{II}$, this method is not intended to be applied to some lock-free data structures that are used in practice.

These temporal-logic based proofs of lock-freedom are quite different from informal lock-freedom proofs of shared data structures in the systems literature (e.g., [7], [9]). The informal argument is that the failure to make progress by a thread is always caused by successful progress in an operation executed by another thread. In this article, we show that this intuitive reasoning can be turned into a formal proof of lock-freedom. To this end, we introduce a quantitative compensation scheme in which a thread that successfully makes progress in an operation has to logically provide resources to other threads to compensate for possible interference it may have caused. Proving that all operations of a data structure adhere to such a compensation scheme is a safety property which can be formalized using minor extensions of modern program logics for fine-grained concurrent programs [14], [15], [16], [17].

We formalize one such extension in this article using CSL. We chose CSL because it has a relatively simple metatheory and can elegantly deal with many challenges arising in the verification of concurrent, pointer-manipulating programs. Parkinson et al. [18] have shown that CSL can be used to derive modular and local safety proofs of non-blocking data structures. The key to these proofs is the identification of a global resource invariant on the shared-data structure that is maintained by each atomic command. However, this technique only applies to safety properties and the authors state that they are "investigating adding liveness rules to separation logic to capture properties such as obstruction/lock/wait-freedom".

We show that it is not necessary to add "liveness rules" to CSL to verify lock-freedom. As in Atkey's separation logic for quantitative reasoning [19] we extend CSL with a predicate for affine tokens to account for, and provide an upper bound on the number of loop iterations in a program. In this way, 
we obtain the first separation logic for total correctness of concurrent programs.

Strengthening the result of Gotsman et al. [13], we first show that lock-freedom can be reduced to the total correctness of concurrent programs in which each thread executes a finite number of data-structure operations. We then prove the total correctness of these programs using our new quantitative reasoning technique and a quantitative resource invariant in the sense of CSL. Thus the proof of the liveness property of being lock-free is reduced to the proof of a stronger safety property. The resulting proofs are simple extensions of memorysafety proofs in CSL and only use standard techniques such as auxiliary variables [20] and read permissions [21].

We demonstrate the practicality of our compensation-based quantitative method by verifying the lock-freedom of Treiber's non-blocking stack $(\S \mathrm{VI})$. We further show that the technique can be used to verify shared-memory data structures such as Michael and Scott's non-blocking queue [7], Hendler et al.'s non-blocking stack with elimination backoff [9], and Michael's non-blocking hazard-pointer data structures [8] (§VII).

Our method is a clean and intuitive modular verification technique that works correctly for shared-memory data structures that have access to thread IDs or the total number of threads in the system (see $\S$ II for details). It can not only be applied to verify total correctness but also to directly prove liveness properties or to verify termination-sensitive contextual refinement. Automation of proofs in concurrent separation logic is an orthogonal issue which is out of the scope of this paper. It would require the automatic generation of loop invariants and resource invariants. Assuming that they are in place, the automation of the proofs can rely on backward reasoning and linear programming as described by Atkey [19].

In summary, we make the following contributions.

1) We introduce a new compensation-based quantitative reasoning technique for proving lock-freedom of nonblocking data structures. ( $(I I I$ and $\S \mathrm{V}$ )

2) We formalize our technique using a novel extension of CSL for total correctness and prove the soundness of this logic. $(\S \mathrm{IV}, \S \mathrm{V}$, and $\S \mathrm{VI})$

3) We demonstrate the effectiveness of our approach by verifying the lock-freedom of Treiber's non-blocking stack (§VI), Michael and Scott's lock-free queue, Hendler et al.'s lock-free stack with elimination backoff, and Michael's lock-free hazard-pointer stack (§VII).

In $\S$ VII, we also discuss how quantitative reasoning can verify the lock-freedom of data structures such as maps and sets, that contain loops that depend on the size of data structures. Finally, in $\S \mathrm{IX}$, we describe other possible applications of quantitative reasoning for proving liveness properties including wait-freedom and starvation-freedom. Appendix II of this article contains all rules of the logic, the semantics, and the full soundness proof.

\section{NON-BLOCKING SYNCHRONIZATION}

Recent years have seen increasing interest in non-blocking data structures [1], [2]: shared-memory data structures that provide operations that are synchronized without using locks and mutual exclusion in favor of performance. A non-blocking data structure is often considered to be correct if its operations are linearizable [3]. Alternatively, correctness can be ensured by an invariant that is maintained by each instruction of the operations. Such an invariant is a safety property that can be proved by modern separation logics for reasoning about concurrent programs [18].

Progress Properties: In this article, we focus on complementary liveness properties that guarantee the progress of the operations of the data structure. There are three different progress properties for non-blocking data structures considered in literature. To define these, assume there is a fixed but arbitrary number of threads that are (repeatedly) accessing a sharedmemory data structure exclusively via the operations it provides. Choose now a point in the execution in which one or more operations has started.

- A wait-free implementation guarantees that every thread can complete any started operation of the data structure in a finite number of steps [4].

- A lock-free implementation guarantees that some thread will complete an operation in a finite number of steps [4]

- An obstruction-free implementation guarantees progress for any thread that eventually executes in isolation [5] (i.e., without other active threads in the system).

Note that these definitions do not make any assumptions on the scheduler. We assume however that any code that is executed between the data-structure operations terminates. If a data structure is wait-free then it is also lock-free [4]. Similarly, lock-freedom implies obstruction-freedom [5]. Wait-free data structures are desirable because they guarantee the absence of live-locks and starvation. However, wait-free data structures are often complex and inefficient. Lock-free data structures, on the other hand, often perform more efficiently in practice. They also ensure the absence of live-locks but allow starvation. Since starvation is an unlikely event in many cases, lock-free data structures are predominant in practice and we focus on them in this paper. However, our techniques apply in principle also to wait-free data structures (see $\S \mathrm{IX}$ ).

Treiber's Stack: As a concrete example we consider Treiber's non-blocking stack [6], a classic lock-free data structure. The shared data structure is a pointer $S$ to a linked list and the operations are push and pop as given in Figure 1.

The operation push $(v)$ creates a pointer $x$ to a new list node containing the data $v$. Then it stores the current stack pointer $S$ in a local variable $t$ and sets the next pointer of the new node $x$ to $t$. Finally it attempts an atomic compare and swap operation $C A S(\& S, t, x)$ to swing $S$ to point to the new node $x$. If the stack pointer $S$ still contains $t$ then $S$ is updated and CAS returns true. In this case, the do-while loop terminates and the operation is complete. If however, the stack pointer $S$ has been updated by another thread so that it no longer contains $t$ then $C A S$ returns false and leaves $S$ unchanged. The do-while loop then does another iteration, updating the new list node to a new value of $S$. The operation pop works similarly to $\operatorname{push}(v)$. If the stack is empty $(t==N U L L)$ then 


\section{Technical Report YALEU/DCS/TR-1476}

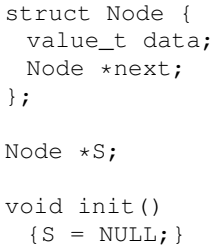

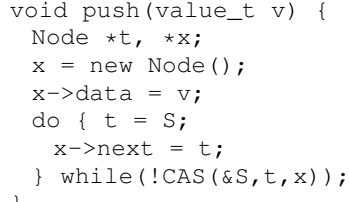

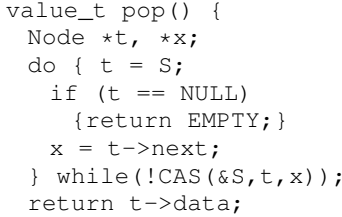

Fig. 1. An implementation of Treiber's lock-free stack as given by Gotsman et al. [13].

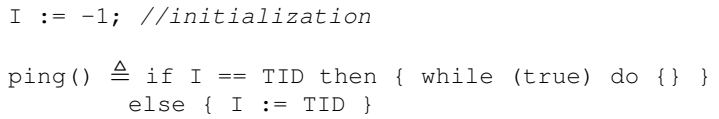

Fig. 2. A shared data structure that shows a limitation of the method of proving lock-freedom that has been introduced by Gotsman et al. [13]. For every $n$, the parallel execution of $n$ ping operations terminates. However, the data structure is not lock-free. (It is based on an idea from James Aspnes.)

pop returns EMPTY. Otherwise it repeatedly tries to update the stack pointer with the successor of the top node using a do-while loop guarded by a CAS.

Treiber's stack is lock-free but not wait-free. If other threads execute infinitely many operations they could prevent the operation of a single thread from finishing. The starvation of one thread is nevertheless only possible if infinitely many operations from other threads succeed by performing a successful CAS. The use of do-while loops that are guarded by CAS operations is characteristic for lock-free data structures.

Lock-Freedom and Termination: Before we verify Treiber's stack, we consider lock-freedom in general. Following an approach proposed by Gotsman et al. [13], we reduce the problem of proving lock-freedom to proving termination of a certain class of programs. Let $D$ be any shared-memory data structure with $k$ operations $\pi_{1}, \ldots, \pi_{k}$. It has been argued [13] that $D$ is lock-free if and only if the following program terminates for every $n \in \mathbb{N}$ and every $o p_{1}, \ldots, o p_{n} \in\left\{\pi_{1}, \ldots \pi_{k}\right\}$ : $O_{n}=\|_{i=1, \ldots, n} o p_{i}$. However, this reduction does not apply to all shared-memory data structures. Many non-blocking data structures have operations that can distinguish different callers, for instance by accessing their thread ID. A simple example is described in Figure 2. The shared data structure consists of an integer $I$ and a single operation ping. If ping is executed twice by the same thread without interference from another thread then the second execution of ping will not terminate. Otherwise, each call of ping immediately returns. As a result, the program $\|_{i=1, \ldots, n}$ ping terminates for every $n$ but the data structure is not lock-free.

We are also aware of a similar example that uses the total number of threads in the system instead of thread IDs. It is in general very common to use these system properties in non-blocking data structures. Three of the five examples in our paper use thread IDs (the hazard pointer stack, the hazard pointer queue, and the elimination-backoff stack).

Consequently, we have to prove a stronger termination property to prove that a data structure is lock-free. Instead of assuming that each client only executes one operation, we assume that each client can execute finitely many operations.
To this end, we define a set of programs $\mathcal{S}^{n}$ that sequentially execute $n$ operations.

$$
\mathcal{S}^{n}=\left\{o p_{1} ; \ldots ; o p_{n} \mid \forall i: o p_{i} \in\left\{\pi_{1}, \ldots, \pi_{k}\right\}\right\}
$$

Let $\mathcal{S}=\bigcup_{n \in \mathbb{N}} \mathcal{S}^{n}$. We now define the set of programs $\mathcal{P}^{m}$ that execute $m$ programs in $\mathcal{S}$ in parallel.

$$
\mathcal{P}^{m}=\left\{\|_{i=1, \ldots, m} s_{i} \mid \forall i: s_{i} \in \mathcal{S}\right\}
$$

Finally, we set $\mathcal{P}=\bigcup_{m \in \mathbb{N}} \mathcal{P}^{m}$. For proving lock-freedom, we rely on the following theorem. By allowing a fixed but arbitrary number of operations per thread we avoid the limitations of the previous approach.

Theorem 1. The data structure $D$ with operations $\pi_{1}, \ldots, \pi_{k}$ is lock-free if and only if every program $P \in \mathcal{P}$ terminates.

Proof. Assume first that $D$ is lock-free. Let $P \in \mathcal{P}$. We prove that $P$ terminates by induction on the number of incomplete operations in $P$, that is, operations that have not yet been started or operations that have been started but have not yet completed. If no operation is incomplete then $P$ immediately terminates. If $n$ operations are incomplete then the scheduler has already or will start an operation by executing one of the threads. By the definition of lock-freedom, some operation will complete independently of the choices of the scheduler. So after a finite number of steps, we reach a point in which only $n-1$ incomplete operations are left. The termination argument follows by induction.

To prove the other direction, assume now that every program $P \in \mathcal{P}$ terminates. Furthermore, assume for the sake of contradiction that $D$ is not lock-free. Then there exists some concurrent program $P_{\infty}$ that only executes operations $o p \in\left\{\pi_{1}, \ldots, \pi_{k}\right\}$ and an execution trace $\mathcal{T}$ of $P_{\infty}$ in which some operations have started but no operation ever completes. It follows that $P_{\infty}$ diverges and $\mathcal{T}$ is therefore infinite. Let $n$ be the number of threads in $P_{\infty}$ and let $s_{i}$ be the sequential program that consists of all operations that have been started by thread $i$ in the execution trace $\mathcal{T}$ in their temporal order. Then the program $\|_{i=1, \ldots, n} s_{i} \in \mathcal{P}^{n}$ can be scheduled to produce the infinite execution trace $\mathcal{T}$. This contradicts the assumption that every program in $\mathcal{P}$ terminates.

\section{Quantitative Reasoning to Prove LOCK-FREEDOM}

A key insight of our work is that for many lock-free data structures, it is possible to give an upper bound on the total number of loop iterations in the programs in $\mathcal{P}(\S I I)$.

To see why, note that most non-blocking operations are based on the same optimistic approach to concurrency. They 
repeatedly try to access or modify a shared-memory data structure until they can complete their operation without interference by another thread. However, lock-freedom ensures that such interference is only possible if another operation successfully makes progress:

In an operation of a lock-free data structure, the failure of a thread to make progress is always caused by successful progress in an operation executed by another thread.

This property is the basis of a novel reasoning technique that we call a quantitative compensation scheme. It ensures that a thread is compensated for loop iterations that are caused by progress-often the successful completion of an operation-in another thread. In return, when a thread makes progress (e.g., completes an operation), it compensates the other threads. In this way, every thread is able to "pay" for its loop iterations without being aware of the other threads or the scheduler.

Consider for example Treiber's stack and a program $P_{n}$ in which every thread only executes one operation, that is, $P_{n}=$ $\|_{i=1, \ldots, n} s_{i}$ and $s_{i} \in\{$ push, pop $\}$. An execution of $P_{n}$ never performs more than $n^{2}$ loop iterations. Using a compensation scheme, this bound can be verified in a local and modular way. Assume that each of the threads has a number of tokens at its disposal and that each loop iteration in the program costs one token. After paying for the loop iteration, the token disappears from the system. Because it is not possible to create or duplicate tokens - tokens are an affine resource - the number of tokens that are initially present in the system is an upper bound on the total number of loop iterations executed.

Unfortunately, the maximum number of loop iterations performed by a thread depends on the choices of the scheduler as well as the number of operations that are performed by the other threads. To still make possible local and modular reasoning, we define a compensation scheme that enables the threads to exchange tokens. Since each loop iteration in $P_{n}$ is guarded by a CAS operation this compensation scheme can be conveniently integrated into the specification of CAS. To this end, we require that (logically) $n-1$ tokens have to be available to execute a $C A S$.

(a) If the CAS is successful then it returns true and (logically) 0 tokens. Thus, the executing thread loses $n-1$ tokens.

(b) If the CAS is unsuccessful then it returns false and (logically) $n$ tokens. Thus, the executing thread gains a token that it can use to pay for its next loop iteration.

The idea behind this compensation scheme is that every thread needs $n$ tokens to perform a data structure operation. One token is used to pay for the first loop iteration and $n-1$ tokens are available during the loop as the loop invariant. If the $C A S$ operation of a thread $A$ is successful (case (a)) then this can cause at most $n-1 C A S$ operations in the other threads to fail. These $n-1$ failed $C A S$ operations need to return one token more than they had prior to their execution (case (b)). On the other hand, the successful thread $A$ does not need its tokens anymore since it will exit the do-while loop. Therefore the $n-1$ tokens belonging to $A$ are passed to the other $n-1$ threads to pay for the worst-case scenario in which this update causes $n-1$ more loop iterations.

If the CAS operation of a thread $A$ fails (case (b)), then some other thread successfully updated the stack (case (a)) and thus provided a token for thread $A$. Since $A$ had $n-1$ tokens before the execution of the $C A S$, it has $n$ tokens after the execution. So thread $A$ can pay a token for the next loop iteration and maintain its loop invariant of $n-1$ available tokens.

In our example program $P_{n}$, there are $n^{2}$ many tokens in the system at the beginning of the execution. So the number of loop iterations is bounded by $n^{2}$ and the program terminates. ${ }^{1}$ More generally, we can use the same local and modular reasoning to prove that every program with $n$ threads such that thread $i$ executes $m_{i}$ operations performs at most $\sum_{1<i<n} m_{i} \cdot n$ loop iterations. Thread $i$ then starts with $m_{i} \cdot n$ tokens.

We will show in the following that this quantitative reasoning can be directly incorporated in total correctness proofs for these programs. We use the exact same techniques (for proving safety properties [18]) to prove liveness properties; namely concurrent separation logic, auxiliary variables, and read permissions. The only thing we add to separation logic is the notion of a token or a resource following Atkey [19].

\section{Preliminary Explanations}

Before we formalize the proof outlined in $\S$ III, we give a short introduction to separation logic, quantitative reasoning, and concurrent separation logic. For the reader unfamiliar with the separation logic extensions of permissions and auxiliary variables, see Appendix I and the relevant literature [20], [21]. Our full logic is defined in Appendix II.

Separation Logic: Separation logic [22], [23] is an extension of Hoare logic [24] that simplifies reasoning about shared mutable data structures and pointers. As in Hoare logic, programs are annotated with Hoare triples using predicates $P, Q, \ldots$ over program states (heap and stack). A Hoare triple $[P] C[Q]$ for a program $C$ is a total-correctness specification of $C$ that expresses the following. If $C$ is executed in a program state that satisfies $P$ then $C$ safely terminates and the execution results in a state that satisfies $Q$.

In addition to the traditional logical connectives, predicates of separation logic are formed by logical connectives that enable local and modular reasoning about the heap. The separating conjunction $P * Q$ is satisfied by a program state if the heap of that state can be split in two disjoint parts such that one sub-heap satisfies $P$ and one sub-heap satisfies $Q$. It enables the safe use of the frame rule

$$
\frac{[P] C[Q]}{[P * R] C[Q * R]} \text { (Frame) }
$$

With the frame rule it is possible to specify only the part of the heap that is modified by the program $C$ (using predicates $P$ and $Q$ ). This specification can then be embedded in a larger proof to state that other parts of the heap are not changed (predicate $R$ ).

\footnotetext{
${ }^{1}$ In fact there are at most $\left(\begin{array}{l}n \\ 2\end{array}\right)$ loop iterations in the worst case. However, the $n^{2}$ bound is sufficient to prove termination.
} 
Quantitative Reasoning: Being based on the logic of bunched implications [25], separation logic treats heap cells as linear resources in the sense of linear logic. It is technically unproblematic to extend separation logic to reason about affine consumable resources too [19]. To this end, the logic is equipped with a special predicate $\diamond$, which states the availability of one consumable resource, or token. The predicate is affine because it is satisfied by every state in which one or more tokens are available. This in contrast to a linear predicate like $E \mapsto F$ that is only satisfied by heaps $H$ with $|\operatorname{dom}(H)|=1$.

Using the separating conjunction $\diamond * P$, it is straightforward to state that two or more tokens are available. We define $\diamond^{n}$ to be an abbreviation for $n$ tokens $\diamond * \ldots * \diamond$ that are connected by the separating conjunction $*$.

Since we use consumable resources to model the terminating behavior of programs, the semantics of the while command are extended such that a single token is consumed, if available, at the beginning of each iteration. Correspondingly, the derivation rule for while commands ensures that a single token is available for consumption on each loop iteration and thus that the loop will execute safely:

$$
\frac{P \wedge B \Longrightarrow P^{\prime} * \diamond \quad I \vdash\left[P^{\prime}\right] C[P]}{I \vdash[P] \text { while } B \text { do } C[P \wedge \neg B]} \text { (While) }
$$

The loop body $C$ must preserve the loop invariant $P$ under the weakened precondition $P^{\prime}$. $C$ is then able to execute under the assumption that one token has been consumed and still restore the invariant $P$, thus making a token available for possible future loop iterations.

The tokens $\diamond$ can be freely mixed with other predicates using the usual connectives of separation logic. For instance, the formula $x \mapsto 10 \vee\left(x \mapsto{ }_{-} * \diamond\right)$ expresses that the heap-cell referred to by the variable $x$ points to 10 , or the heap-cell points to an arbitrary value and a token is available. Together with the frame rule, the tokens enable modular reasoning about quantitative resource usage.

Concurrent Separation Logic: Concurrent separation logic (CSL) is an extension of separation logic that is used to reason about concurrent programs [14]. The idea is that shared memory regions are associated with a resource invariant. Each atomic block that modifies the shared region can assume that the invariant holds at the beginning of its execution and must ensure that the invariant holds at the end of the atomic block.

The original presentation of CSL [14] uses conditional critical regions (CCRs) for shared variables. In this article, we follow Parkinson et al. [18] and assume a global shared region with one invariant so as to simplify the syntax and the logic. An extension to CCRs is possible. For predicates $I, P$, and $Q$, the judgment $I \vdash[P] C[Q]$ states that under the global resource invariant $I$, in a state where $P$ holds, the execution of the concurrent program $C$ is safe and terminates in a state that satisfies $Q$.

Concurrent execution of $C_{1}$ and $C_{2}$ is written $C_{1} \| C_{2}$. We assume that shared variables are only accessed within atomic regions using the command atomic $(C)$ and that atomic blocks are not nested. An interpretation of the resource invariant $I$ is that it specifies a part of the heap owned by the shared region. The logical rule АТОM for the command atomic transfers the ownership of this heap region to the executing thread.

$$
\frac{e m p \vdash[P * I] C[Q * I]}{I \vdash[P] \text { atomic }\{C\}[Q]} \text { (Атом) }
$$

Because the atomic construct ensures mutual exclusion, it is safe to share $I$ between two programs that run in parallel. Pre- and post-conditions of concurrent programs are however combined by use of the separating conjunction ${ }^{2}$ :

$$
\frac{I \vdash\left[P_{1}\right] C_{1}\left[Q_{1}\right] \quad I \vdash\left[P_{2}\right] C_{2}\left[Q_{2}\right]}{I \vdash\left[P_{1} * P_{2}\right] C_{1} \| C_{2}\left[Q_{1} * Q_{2}\right]}(\mathrm{PAR})
$$

Most of the other rules of sequential separation logic can be used in CSL by just adding the (unmodified) resource invariant $I$ to the rules. The invariant is only used in the rule ATOM.

A technical detail that is crucial for the soundness of the classic rule for conjunction [24] is that we require the resource invariant $I$ to be precise [14] with respect to the heap [26]. This means that, for a given heap $H$ and stack $V$, there is at most one sub-heap $H^{\prime} \subseteq H$ such that the sate $\left(H^{\prime}, V\right)$ satisfies $I$. All invariants we use in this article are precise. Note that precision with respect to the resource tokens $\diamond$ is not required since they are affine and not linear entities.

\section{Formalized Quantitative Reasoning}

In the following, we show how quantitative concurrent separation logic can be used to formalize the quantitative compensation scheme that we exemplified with Treiber's nonblocking stack in $\S I I I$. The most important rules of this logic are described in $\S I V$. The logic is formally defined and proved sound in Appendix II.

Before we verify realistic non-blocking data structures, we describe the formalized quantitative reasoning for a simple producer and consumer example.

Producer and Consumer Example: In the example in Figure 3, we have a heap location $B$ that is shared between a number of producer and consumer threads. A producer checks whether $B$ contains the integer 0 (i.e., $B$ is empty). If so then it updates $B$ with a newly produced value and terminates. Otherwise, it leaves $B$ unchanged and terminates. A consumer checks whether $B$ contains a non-zero integer (i.e, $B$ is nonempty). If so then it consumes the integer, sets the contents of $B$ to zero, and loops to check if $B$ contains a new value to consume. If $B$ contains 0 then the consumer terminates.

If we verify this program using our quantitative separation logic then we prove that the number of tokens specified by the precondition is an upper bound on the number of loop iterations of the program. Since the number of specified tokens is always finite, we have thus proved termination.

The challenge in the proof is that the loop iterations of the operation Consumer depend on the scheduler and on the number of Producer operations that are executed in parallel. However, it is the case that a program that uses $n$ Consumer

\footnotetext{
${ }^{2}$ We omit the variable side-conditions here for clarity. They are included in the full set of derivation rules in Appendix II.
} 


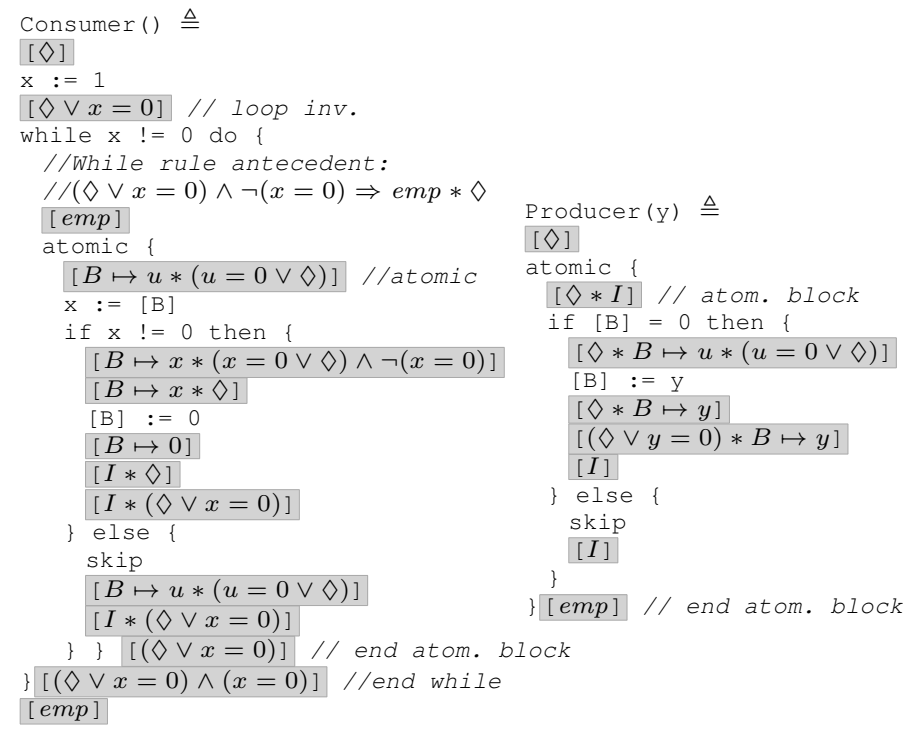

Fig. 3. A lock-free data structure $B$ with the operations Consumer and Producer. The operation Consumer terminates if finitely many Producer operations are executed in parallel. The verification of lock-freedom and memory safety uses a compensation scheme and quantitative concurrent separation logic.

operations and $m$ Producer operations performs at most $n+m$ loop iterations. We can prove this claim using our quantitative separation logic by deriving the following specifications.

$$
I \vdash[\diamond] \text { Consumer() }[e m p] \text { and } I \vdash[\diamond] \operatorname{Producer}(y)[e m p]
$$

However, the modular and local specifications of these operations only hold in an environment in which all programs adhere to a certain policy. This policy can be expressed as a resource invariant $I$ in the sense of concurrent separation logic. Intuitively, $I$ states that the shared memory location $B$ is read-writable, and either is empty $(B=0)$ or there is a token $\diamond$ available. We define

$$
I \triangleq \exists u . B \mapsto u *(u=0 \vee \diamond) .
$$

Now we can read the specifications of Consumer and Producer as follows. The token $\diamond$ in the precondition of Consumer is used to pay for the first loop iteration. More loop iterations are only possible if some producer updated the contents of heap location $B$ to a non-zero integer $v$ before the execution of the atomic block of Consumer. We then rely on the fact that the producer respected the resource invariant $I$. If $B \mapsto u$ and $u \neq 0$ then the only possibility of maintaining $I$ is by providing a token $\diamond$. The operation Consumer then updates $B$ to zero and can thus establish the invariant $I$ without using a token. So the token in the invariant becomes available to pay for the next loop iteration. Figure 3 contains an outline of the proof for Producer and Consumer. Note that our proof also verifies memory safety.

From Local Proofs to Lock-Freedom: Using the derived specifications of the operations and the frame rule, we inductively prove $I \vdash\left[\diamond^{k}\right] o p_{1} ; \ldots ; o p_{k}[e m p]$ where each $o p_{i}$ is a Consumer or Producer operation. In other words, we have then proved $\left[\diamond^{k}\right] s[e m p]$ for all $s \in \mathcal{S}^{k}$ (recall the definition from $\S$ II). Let now $s_{i} \in \mathcal{S}^{m_{i}}$ for $1 \leq i \leq n$. Using the rule
PAR, we can then prove for $m=\sum_{i=1, \ldots, n} m_{i}$ that

$$
I \vdash\left[\diamond^{m}\right] \|_{i=1, \ldots, n} s_{i}[e m p] .
$$

This shows that the program $\|_{i=1, \ldots, n} s_{i}$ performs at most $m+1$ loop iterations (one token can be present in the resource invariant $I$ ) when it is executed. Following the discussion in $\S I I$, this proves that every program $p \in \mathcal{P}$ terminates and that ( $B$; Producer, Consumer) is a lock-free data structure.

Similarly, we can in general derive a termination proof for every program in $\mathcal{P}$ from such specifications of the operations of a data structure. Assume that a shared-memory data structure $\left(S ; \pi_{1}, \ldots, \pi_{k}\right)$ is given. Assume furthermore that we have verified for all $1 \leq i \leq k$ the specification $I(n) \vdash\left[\diamond^{f(n)} *\right.$ $P] \pi_{i}[P]$. The notations $I(n)$ and $f(n)$ indicate that the proof can use a meta-variable $n$ which ranges over $\mathbb{N}$. However, the proof is uniform for all $n$. Additionally, $P$ might contain a variable $t i d$ for the thread ID. From this specification follows already the lock-freedom of $S$. To see why, we can argue as in the producer-consumer example. First, it follows for every $n$ and $s \in \mathcal{S}^{m}$ that $I(n) \vdash\left[\diamond^{m \cdot f(n)} * P\right] s[P]$. Second, a loop bound for $p=\|_{i=1, \ldots, n} s_{i} \in \mathcal{P}^{n}$ with $s_{i} \in \mathcal{S}^{m_{i}}$ is derived as follows. We use the rule PAR to prove for $m=$ $\sum_{i=1, \ldots, n} m_{i} \cdot f(n)$ that

$$
I(n) \vdash\left[\diamond^{m} * \underset{0 \leq t i d<n}{\circledast} P(t i d)\right] p[\underset{0 \leq t i d<n}{\circledast} P(t i d)] .
$$

Thus every $p \in \mathcal{P}$ terminates and according to the proof in $\S I$, the data structure $\left(S ; \pi_{1}, \ldots, \pi_{k}\right)$ is lock-free.

\section{LOCK-FrEEdOM OF TREIBER'S STACK}

We now formalize the informal proof of the lock-freedom of Treiber's stack that we described in $§$ III. In Appendix III, we outline how the proof can be easily extended to also verify memory safety. Figure 4 shows the implementation of Treiber's stack in the while language we use in this article.

Each thread that executes push or pop operations can be in one of two states. It either has some expectation on the contents of the shared data structure $S$ (critical state) or it does not have any expectation (non-critical state). More concretely, a thread is in a critical state if and only if it is executing a push or pop operation and is in between the two atomic blocks in the while loop. The thread then expects that $t=[S]$. The resource invariant that we will formalize in quantitative CSL can be described as follows.

For each thread $T$ in the system one of the following holds.

(1) The thread $T$ is in a critical state and its expectation on the shared data structure is true. (2) The thread $T$ is in a critical state and some other thread provided $T$ with a token. (3) The thread $T$ is in a non-critical state.

To formalize this invariant, we have to expose the local assumption of the threads $(t=[S])$ to the global state. This is why we use the auxiliary array $A$. If the thread with the thread ID $t i d$ is in a critical state then $A[t i d]$ contains the value of its local variable $t$. Otherwise $A[t i d]$ contains 0 . Similarly, we have a second auxiliary array $C$ such that $C[t i d]$ contains a non-zero integer if and only if the thread with ID tid is in a 


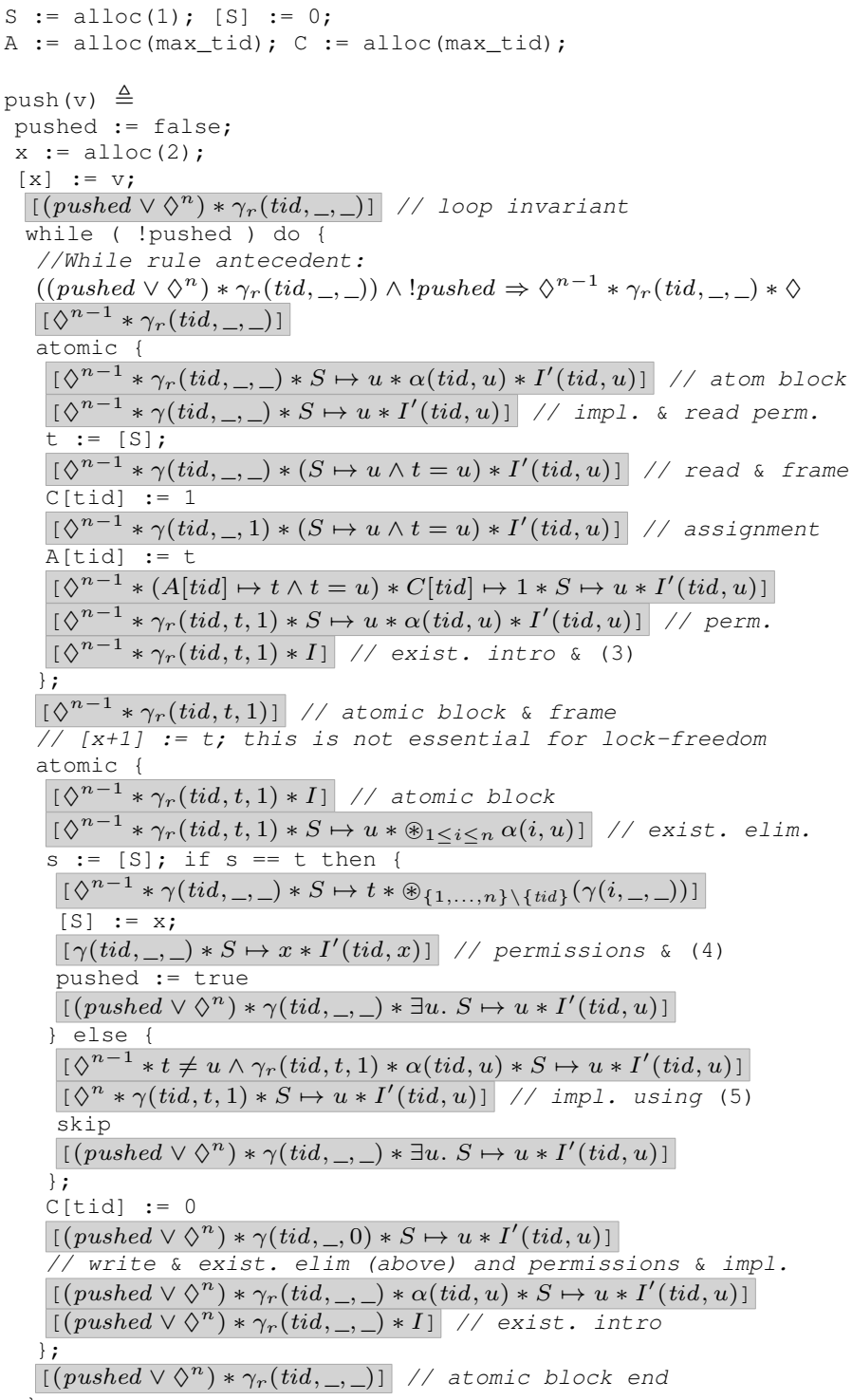

Fig. 4. An implementation of the push operation of Treiber's lock-free stack in our language and the verification of the while loop. The CAS operation is implemented using an atomic block that updates the local variable pushed The auxiliary array $A$ contains in $A[t i d]$ the value of the local variable $t$ of the thread with ID tid or zero if the thread does not assume $t=[S]$. The loop invariant pushed $\vee \diamond^{n}$ states that either the new element $x$ has been pushed to the stack $S$ or there are $n$ tokens available. The predicates $\gamma$ and $\gamma_{r}$ are defined in (1).

critical state. As shown in Figure 4, the arrays $A$ and $C$ are never used on the right-hand side of an assignment and are only updated in the two atomic blocks of each operation.

Let $n$ be the number of threads in the system. We define

$$
\begin{gathered}
I \triangleq \exists u . S \mapsto u * \underset{0 \leq i<n}{\circledast} \alpha(i, u) \\
\alpha(i, u) \triangleq \exists a, c . C[i] \stackrel{r}{\mapsto} c * A[i] \stackrel{r}{\mapsto} a *(c=0 \vee a=u \vee \diamond)
\end{gathered}
$$

The resource invariant $I$ states that the shared region has a full permission for the heap location $S$ that points to the value $u$. Additionally, the predicate $\alpha(i, u)$ states for each thread $i$ that the shared region has read permissions for $C[i]$ and $A[i]$; and that thread $i$ is in a non-critical section $(c=0)$, that the local variable $t$ contains the value $[S](a=u)$, or that there is a token $\diamond$ available.

We use read permissions since threads need access to the local predicate $A[t i d] \stackrel{r}{\mapsto} t$ at some point to infer that $A[t i d]$ contains the value of the local variable $t$. This relation of the local variable $t$ with the array $A$ is the only technical difficulty in the proof. Just as in safety proofs, we can now use the rules of our quantitative concurrent separation logic to verify the following Hoare triples.

$$
\begin{aligned}
& I \vdash\left[\gamma_{r}\left(t i d,,_{-},{ }_{-}\right) * \diamond^{n}\right] \operatorname{push}(v)\left[\gamma_{r}\left(t i d,,_{-},-\right)\right] \\
& \left.I \vdash\left[\gamma_{r}\left(t i d,,_{-},,_{-}\right) * \diamond^{n}\right] \quad \text { pop( }\right) \quad\left[\gamma_{r}\left(t i d,,_{-},-_{-}\right)\right]
\end{aligned}
$$

Where $\gamma$ and $\gamma_{r}$ are defined as:

$$
\begin{aligned}
\gamma(t, a, c) & \triangleq A[t] \mapsto a * C[t] \mapsto c \\
\gamma_{r}(t, a, c) \triangleq A[t] \mapsto a r & \mapsto * C[t] \mapsto c
\end{aligned}
$$

Thus, the execution of any operation requires $n$ tokens and read permission to the heap locations $A[t i d]$ and $C[t i d]$. After execution, the tokens are consumed and we are left with the read permissions. We use the following abbreviation for parts of the invariant $I$ that are not needed in the local proofs.

$$
I^{\prime}(j, u) \triangleq \underset{i \in\{0, \ldots, n-1\} \backslash\{j\}}{\circledast} \alpha(i, u)
$$

We have for all values $u$ and $j \in\{0, \ldots, n-1\}$ that

$$
\begin{gathered}
I=\exists u . S \mapsto u * \alpha(j, u) * I^{\prime}(j, u) \\
\diamond^{n-1} \underset{i \in\{0, \ldots, n-1\} \backslash\{j\}}{\circledast}\left(\gamma_{r}\left(i,,_{-}\right)\right) \Longrightarrow I^{\prime}(j, u) \\
t \neq u \wedge \gamma_{r}(j, t, 1) * \alpha(j, u) \Longrightarrow \diamond * \gamma(j, t, 1)
\end{gathered}
$$

Using these assertions, the verification of push and pop is a straightforward application of the rules of our logic. Figure 4 describes the main part-the while loop-of the proof of push. The loop invariant pushed $\vee \nabla^{n}$ states that either the new element $x$ has been pushed onto the stack $S$ or there are $n$ tokens available. In the first atomic block we leave the assumptions $I^{\prime}(t i d, u)$ of the other thread untouched and just establish $A[t i d] \stackrel{r}{\mapsto} t * C[t i d] \stackrel{\mapsto}{\mapsto} 1$.

The key aspect of the proof is the second atomic block which corresponds to the CAS operation in the original code. In the if case, we possibly break the assumptions of the other threads $([S]:=x)$. Then we have to use $n-1$ tokens and implication (4) to re-establish $I^{\prime}(t i d, u)$. Since the variable pushed is set to true, we can maintain the loop invariant without using another token. In the else case we use the inequality $t \neq u$ and implication (5) to derive the loop invariant. Finally, we re-establish $\alpha(t i d, u)$ using $C[t i d] \mapsto 0$.

The verification of the while loop of pop is similar. By applying the proof from the end of $\S \mathrm{V}$ to the specifications of push and pop, we have then proved the lock-freedom of Treiber's stack.

An interesting aspect of the proof is that it is not essential for a thread to know the entire resource invariant $I$. The only part that is needed is the implication $S \mapsto \mapsto_{-} * \diamond^{n} * \circledast_{0 \leq i<n} A[i] \stackrel{r}{\mapsto}$ - $\Longrightarrow I$. This can be used to make the assumptions $A(i)$ of the threads on the global data structure abstract. The implication $S \mapsto{ }_{-} * \diamond^{n} * \circledast_{0 \leq i<n} A[i] \stackrel{r}{\mapsto}{ }_{-} \Longrightarrow$ 
$\exists u . S \mapsto \mapsto_{-} * \circledast_{0<i<n}\left(\left(A[i] \stackrel{r}{\mapsto}{ }_{-} * \diamond\right) \vee A(i, u)\right)$ holds for all predicates $A(i, u)$. A natural candidate for such an abstraction is (concurrent) abstract predicates [27], [28]. However, such an abstraction is not needed for our goal of verifying non-blocking data structures in this paper.

\section{Advanced Lock-Free Data Structures}

In this section we investigate how our quantitative proof technique can be used to prove the lock-freedom of more complex shared-memory data structures.

In many cases, it is possible to derive a bound on the total number of loop iterations like we do for Treiber's stack. Table 5 gives an overview of our findings. It describes for several different data structures the number $t(n)$ of tokens that are needed per operation in a system with $n$ threads. The derived loop bound on a system with $n$ threads that executes $m$ operations is then $t(n) * m$. In the hazard-pointer data structures, the natural number $\ell$ is a fixed global parameter of the data structure. The details are discussed in the following.

Michael and Scott's Non-Blocking Queue: Michael and Scott's non-blocking queue [7] implements a FIFO queue using a linked list with pointers to the head and the tail. New nodes are inserted at the tail and nodes are removed from the head.

To implement the queue in a lock-free way, the insert operation can leave the data structure in an apparently inconsistent state: The new node is inserted at the tail using a CAS-guarded loop, similar to Treiber's stack. The pointer to the tail is then updated by a second CAS operation, allowing other threads to access the data structure with an inaccurate tail pointer.

To deal with this problem, the operations of the queue maintain the invariant that the tail pointer points to the last or second-to-last node during the execution and to the last node after the execution of the operation. To maintain this invariant, each CAS-guarded loop checks if the tail pointer points to a node whose next pointer is Null. In this case, the tail pointer is up to date and the current iteration of the while loop can continue. Otherwise, the tail pointer is updated to point to the last node of the list and the while loop is restarted.

To prove the lock-freedom of Michael and Scott's queue, we extend the invariant $I$ that we used in the verification of Treiber's stack with an additional condition: The next pointer of the node pointed to by the tail pointer is Null or there is a token that can be used to pay for an additional loop iteration.

$$
\begin{aligned}
& \exists u, t, w \text {.heap } \mapsto u * \text { tail } \mapsto t * \text { tail }+1 \mapsto w * \\
& \underset{0 \leq i<n}{\circledast} \beta(i, u, t) *(w=n i l \vee \diamond)
\end{aligned}
$$

The formulas $\beta(i, u, t)$ are analogous to the formulas $\alpha(i, u)$ in the invariant that we used for the verification of Treiber's stack. With this invariant in a system with $n$ threads, we can verify the operations of the queue using $n+1$ tokens in the respective preconditions.

Hazard Pointers: A limitation of Treiber's non-blocking stack is that it is only sound in the presence of garbage collection. This is due to the ABA problem (see for instance [8]) which appears in many algorithms that use compare-and-swap operations: Assume that a shared location which contains $A$

\begin{tabular}{lc}
\hline Data Structure & Tokens per Operation \\
\hline Treiber's Stack [6] & $n$ \\
Michael and Scott's Queue [7] & $n+1$ \\
Hazard-Pointer Stack [8] & $n+(\ell \cdot n)$ \\
Hazard-Pointer Queue [8] & $(n+1)+(\ell \cdot n)$ \\
Elimination-Backoff Stack [9] & $n(n+1)$
\end{tabular}

Fig. 5. Quantitative reasoning for popular non-blocking data structures. The table shows the number $t(n)$ of tokens that are needed per operation in a system with $n$ threads. The derived loop bound on a system with $n$ threads that executes $m$ operations is then $t(n) * m$. The natural number $\ell$ is a fixed global parameter of the data structure.

is read by a thread $t_{1}$. Then thread $t_{2}$ gets activated by the scheduler, modifies the shared location to $B$, and then back to $A$. Now thread $t_{1}$ gets activated again, falsely assumes that the shared data has not been changed, and continues with its operation. The result can be a corrupted shared data structure, invalid memory access or an incorrect return value.

Michael [8] proposes hazard pointers to enable the safe reclamation of memory while maintaining the lock-freedom of non-blocking data structures. The idea is to introduce a global array that contains for each thread a number of pointers (in most cases just one) to data nodes that are currently in use by the thread. Additionally, each thread stores a local list of pointers that it wants to remove from the shared data structure (for instance by using pop in the case of a stack). After each successful removal of a node a thread checks if this local list has reached a fixed length threshold. If so, it checks the hazard pointers of each other thread to ensure that the pointers are not in use before reclaiming the space.

The use of hazard pointers does not affect the global resource invariants that we use in our quantitative verification technique. The reason is that hazard pointers only affect parts of the operations that are outside the loops guarded by CAS operations. Moreover, the worst-case number of loop iterations in this additional code can be easily determined: It is the maximal length $\ell$ of the local list multiplied with the maximal number of threads in the system.

For Treiber's stack with hazard pointers, the specifications of push and pop are:

$$
\left.\begin{array}{rcc}
I \vdash\left[\gamma_{r}\left(t i d,,_{-},{ }_{-}\right) * \diamond^{n}\right] & \text { push(v) } & {\left[\gamma_{r}\left(t i d,,_{-},{ }_{-}\right)\right.}
\end{array}\right]
$$

Where $\gamma_{r}$ is defined as in (1). The invariant $I$ is the same as in the specification of the version without hazard pointers.

Elimination Backoff: To improve the performance of Treiber's non-blocking stack in the presence of high contention, one can use an elimination backoff scheme [9]. The idea is based on the observation that a push operation followed by a pop results in a stack that is identical to the initial stack. So, if a stack operation fails because of the interference of another thread then the executing thread does not immediately retry the operation. Instead, it checks if there is another thread that is trying to perform a complementary operation. In this case, the two operations can be eliminated without accessing the 
stack at all: The two threads use a different shared-memory cell to transfer the stack element.

Our method can also be used to prove that Hendler et al's elimination-backoff stack [9] is lock-free. The main challenge in the proof is that the push and pop operations consist of two nested loops guarded by CAS operations. Assume again a system with $n$ threads. The inner loop can be treated as in Treiber's stack using $n$ tokens in the precondition and 0 tokens in the postcondition. As a result, the number of tokens needed for an iteration of the outer loop is $n+1$. That means that a successful thread needs to transfer $(n-1) \cdot(n+1)=n^{2}-1$ tokens to the other threads to account for additional loop iterations in the other threads. Given this, we can verify the elimination-backoff stack using $n^{2}$ tokens in the precondition. Technically, we need an invariant of the form $I * J$, where $I$ is an invariant like in Treiber's stack (for the inner loop) and $J$ is like $I$ but with every token $\diamond$ replaced by $\diamond^{n}$. More details on the verification can be found in Appendix IV.

Non-Blocking Maps and Sets: Quantitative compensation schemes can also be used to prove the lock-freedom of nonblocking maps and sets (e.g., [29], [30]).

As in other lock-free data structures, interference in map and set operations only occurs when another thread makes progress. For example, in Harris' non-blocking linked list [29], a thread will make an additional traversal (of the list) only if another thread causes interference by making a successful traversal. The number of these unsuccessful traversals can be bounded using a quantitative compensation scheme, as in our previous examples.

The number of loop iterations within each list traversal also depends on the length of the list. Nevertheless, it is possible to prove an upper bound on the number of loop iterations executed by programs in $\mathcal{P}$, since each of the $n$ threads executes a fixed number $m_{i}$ of operations. Thus the total number of operations is bounded by $m=\sum_{i=1, \ldots, n} m_{i}$. In many important sharedmemory data structures, such as lists or maps, $m$ (or a function of $m$ ) constitutes an upper bound on the size of the shared data structure. One can then use this bound to prove an upper bound on the number of loop iterations by introducing $\diamond^{m}$ in the global resource invariant. Like Atkey [19], we can use ideas from amortized resource analysis [31] to deal with variablesize data structures. By assigning tokens to each element of a data structure we derive bounds that depend on the size of the data structure without explicitly referring to its size. For instance, an inductive list-predicate that states that $k \cdot|\ell|$ tokens are available, where $\ell$ is the list pointed to by $u$ can be defined as follows.

$$
\begin{aligned}
\operatorname{LSeg}^{\prime}(x, y, k) \Leftrightarrow & (x=y \wedge e m p) \vee \\
& \left(\exists v, z x \mapsto v * x+1 \mapsto z * \operatorname{LSeg}^{\prime}(z, y, k) * \diamond^{k}\right)
\end{aligned}
$$

\section{Related Work}

There is a large body of research on verifying safety properties and partial correctness of non-blocking data structures. See for instance [18], [32], [33] and the references therein. This work deals with the verification of the complementary liveness property of being lock-free, which in comparison has received little attention.

Colvin and Dongol [10], [34] use manually-derived global well-founded orderings and temporal logic to prove the lockfreedom of Treiber's stack [6], Michael and Scott's queue [7], and a bounded array-based queue. Their technique is a nonmodular whole program analysis of the most general client of the data structure. It is unclear if the approach applies to data-structure operations with nested loops. In contrast, our method is modular, can handle with nested loops, and does not require temporal reasoning.

Petrank et al. [11] endeavor to reduce lock-freedom to a safety property by introducing the more restrictive concept of bounded lock-freedom. It states that, in a concurrent program, there has to be progress after at most $k$ steps, where $k$ depends on the input size of the program but not on the number of threads in the system. They verify bounded lock-freedom with a whole program analysis using temporal logic and the model checker Chess. The technique is demonstrated by verifying a simple concurrent program that uses Treiber's stack. Our compensation-based quantitative reasoning does not provide an explicit bound on the steps between successful operations but rather a global bound on the number of loop iterations in the system. Additionally, our bound depends on the number of threads in the system and not on the size of the input. A conceptual difference of our work is that we prove the lockfreedom of a given data structure as opposed to the verification of a specific program. Moreover, our proofs are local and modular, and not a whole program analysis. We also show that compensation-based reasoning works for many advanced lock-free data structures.

Gotsman et al. [13] reduce lock-freedom proofs to termination proofs of programs that execute $n$ data structure operations in parallel. They then prove termination using separation logic and temporal rely-guarantee reasoning by layering liveness reasoning on top of a circular safety proof. Using several tools and manually formulating appropriate proof obligations, they are able to automatically verify the lock-freedom of involved algorithms such as Hendler et al.'s non-blocking stack with elimination backoff [9]. While these automation results are very impressive, the used reduction to termination is not intended to be applied to shared data structures that use thread IDs or other system information (see $\S \mathrm{II}$ for details). In comparison, our compensation reasoning does not restrict the use of thread IDs or other system information. According to the authors (personal communication), the termination proofs of [13] would also work for a modification of the reduction that we used here.

Tofan et al. [12] describe a fully-mechanized technique based on temporal logic and rely-guarantee reasoning that is similar to the work of Gotsman et al. However, they assume weak fairness of the scheduler while we do not pose any restriction on the scheduler. Kobayashi and Sangiorgi [35] propose a type system that proves lock-freedom for programs written in the $\pi$-calculus. The target language and examples seem however to be quite different from the programs we prove lock-free in this article. 


\section{CONClusion}

We have shown that lock-freedom proofs of shared-memory data structures can be reduced to safety proofs in concurrent separation logic (CSL). To this end, we proposed a novel quantitative compensation scheme which can be formalized in CSL using a predicate $\diamond$ for affine tokens. While similar logics have been used to verify the resource consumption of sequential programs [19], this is the first time that a quantitative reasoning method has been used to verify liveness properties of concurrent programs.

In the future, we plan to investigate the extent to which quantitative reasoning can be applied to other liveness properties of concurrent programs. The quantitative verification of wait-freedom seems to be similar to the verification of lockfreedom if we require that tokens cannot be transferred among the threads. Obstruction-freedom might require the creation of tokens in case of a conflict. We also plan to adapt our method to reason about progress properties of locking data structures, such as fairness and starvation-freedom. These properties are more challenging to verify with our quantitative method since they rely on a fair scheduler, whereas non-blocking algorithms do not. To enable such proofs, we plan to extend our compensation scheme to include the behavior of the scheduler.

Ultimately, we envision integrating our compensation-based proofs into a logic for termination-sensitive contextual refinement.

\section{ACKNOWLEDGMENTS}

We thank James Aspnes and Alexey Gotsman for helpful discussions. This research is based on work supported in part by DARPA grants FA8750-10-2-0254 and FA8750-12-2-0293, ONR grant N000141210478, and NSF grants 0910670 and 0915888 and 1065451 . The U.S. Government is authorized to reproduce and distribute reprints for Governmental purposes notwithstanding any copyright notation thereon. Any opinions, findings, and conclusions contained in this document are those of the authors and do not reflect the views of these agencies.

\section{REFERENCES}

[1] W. N. Scherer III, D. Lea, and M. L. Scott, "Scalable Synchronous Queues," Commun. ACM, vol. 52, no. 5, pp. 100-111, 2009.

[2] N. Shavit, "Data Structures in the Multicore Age," Commun. ACM, vol. 54, no. 3, pp. 76-84, 2011.

[3] M. P. Herlihy and J. M. Wing, "Linearizability: A Correctness Condition for Concurrent Objects," ACM Trans. Program. Lang. Syst., vol. 12, no. 3, pp. 463-492, 1990

[4] M. Herlihy, "Wait-Free Synchronization," ACM Trans. Program. Lang. Syst., vol. 13, no. 1, pp. 124-149, 1991.

[5] M. Herlihy, V. Luchangco, and M. Moir, "Obstruction-Free Synchronization: Double-Ended Queues as an Example," in 23rd Int. Conf. on Distributed Comp. Systems (ICDCS'03), 2003.

[6] R. K. Treiber, "Systems Programming: Coping with Parallelism," IBM Almaden Research Center, Tech. Rep. RJ 5118, 1986.

[7] M. M. Michael and M. L. Scott, "Simple, Fast, and Practical NonBlocking and Blocking Concurrent Queue Algorithms," in 15th Symp. on Principles of Distributed Computing (PODC'96), 1996, pp. 267-275.

[8] M. M. Michael, "Hazard Pointers: Safe Memory Reclamation for LockFree Objects," IEEE Trans. Parallel Distrib. Syst., vol. 15, no. 6, pp. 491-504, 2004.

[9] D. Hendler, N. Shavit, and L. Yerushalmi, "A Scalable Lock-Free Stack Algorithm," in 16th ACM Symposium on Parallelism in Algorithms and Architectures (SPAA'04), 2004, pp. 206-215.
[10] R. Colvin and B. Dongol, "Verifying Lock-Freedom Using Well-Founded Orders," in Theoretical Aspects of Computing - 4th International Colloquium (ICTAC'07), 2007, pp. 124-138.

[11] E. Petrank, M. Musuvathi, and B. Steesngaard, "Progress Guarantee for Parallel Programs via Bounded Lock-Freedom," in Conf. on Prog. Lang. Design and Impl. (PLDI'09), 2009, pp. 144-154.

[12] B. Tofan, S. Bäumler, G. Schellhorn, and W. Reif, "Temporal Logic Verification of Lock-Freedom," in Mathematics of Prog. Construction, 10th Int. Conf., (MPC'10), 2010, pp. 377-396.

[13] A. Gotsman, B. Cook, M. Parkinson, and V. Vafeiadis, "Proving that Non-Blocking Algorithms Don't Block," in 36th Symp. on Principles of Prog. Lang. (POPL'09), 2009, pp. 16-28.

[14] P. W. O'Hearn, "Resources, concurrency, and local reasoning," Theor Comput. Sci., vol. 375, no. 1-3, pp. 271-307, 2007.

[15] V. Vafeiadis and M. J. Parkinson, "A Marriage of Rely/Guarantee and Separation Logic," in Concurrency Theory, 18th Int. Conference (CONCUR'07), 2007, pp. 256-271.

[16] X. Feng, "Local Rely-Guarantee Reasoning," in 36th Symp. on Principles of Prog. Langs. (POPL'09), 2009, pp. 315-327.

[17] T. Dinsdale-Young, M. Dodds, P. Gardner, M. J. Parkinson, and V. Vafeiadis, "Concurrent Abstract Predicates," in Object-Oriented Programming, 24th European Conf. (ECOOP'10), 2010, pp. 504-528.

[18] M. Parkinson, R. Bornat, and P. O'Hearn, "Modular Verification of a Non-Blocking Stack," in 34th Symp. on Principles of Prog. Lang. (POPL'07), 2007, pp. 297-302.

[19] R. Atkey, "Amortised Resource Analysis with Separation Logic," in 19th European Symposium on Programming (ESOP'10), 2010, pp. 85-103.

[20] S. S. Owicki and D. Gries, "Verifying Properties of Parallel Programs: An Axiomatic Approach," Commun. ACM, vol. 19, no. 5, pp. 279-285, 1976.

[21] R. Bornat, C. Calcagno, P. O'Hearn, and M. Parkinson, "Permission Accounting in Separation Logic," in 32nd Symp. on Principles of Prog. Lang. (POPL'05), 2005, pp. 259-270.

[22] S. S. Ishtiaq and P. W. O'Hearn, "BI as an Assertion Language for Mutable Data Structures," in 28th Symp. on Principles of Prog. Lang. (POPL'01), 2001, pp. 14-26.

[23] J. C. Reynolds, "Separation Logic: A Logic for Shared Mutable Data Structures," in 17th IEEE Symp on Logic in Computer Science (LICS'02), 2002, pp. 55-74.

[24] C. A. R. Hoare, "An Axiomatic Basis for Computer Programming," Commun. ACM, vol. 12, no. 10, pp. 576-580, 1969.

[25] P. W. O'Hearn and D. J. Pym, "The Logic of Bunched Implications," Bulletin of Symbolic Logic, vol. 5, no. 2, pp. 215-244, 1999.

[26] V. Vafeiadis, "Concurrent Separation Logic and Operational Semantics," Electr. Notes Theor. Comput. Sci., vol. 276, pp. 335-351, 2011.

[27] M. J. Parkinson and G. M. Bierman, "Separation Logic and Abstraction," in 32nd Symp. on Principles of Prog. Lang. (POPL'05), 2005, pp. 247258.

[28] T. Dinsdale-Young, M. Dodds, P. Gardner, M. J. Parkinson, and V. Vafeiadis, "Concurrent Abstract Predicates," in Object-Oriented Programming, 24th European Conf. (ECOOP'10), 2010, pp. 504-528.

[29] T. L. Harris, "A Pragmatic Implementation of Non-blocking LinkedLists," in 15th International Conf. on Distributed Computing (DISC'01), 2001, pp. 300-314.

[30] M. Greenwald, "Non-blocking Synchronization and System Design," Ph.D. dissertation, Stanford University, 1999, tR STAN-CS-TR-99-1624.

[31] M. Hofmann and S. Jost, "Static Prediction of Heap Space Usage for First-Order Functional Programs," in 30th Symp. on Principles of Prog. Lang. (POPL'03), 2003, pp. 185-197.

[32] V. Vafeiadis, "Modular Fine-Grained Concurrency Verification," Ph.D dissertation, University of Cambridge, 2007, tR UCAM-CL-TR-726.

[33] M. Fu, Y. Li, X. Feng, Z. Shao, and Y. Zhang, "Reasoning about Optimistic Concurrency Using a Program Logic for History," in Concurrency Theory, 21th Int. Conference (CONCUR'10), 2010, pp. 388-402.

[34] R. Colvin and B. Dongol, "A General Technique for Proving LockFreedom,” Sci. Comput. Program., vol. 74, no. 3, pp. 143-165, 2009.

[35] N. Kobayashi and D. Sangiorgi, "A Hybrid Type System for LockFreedom of Mobile Processes," ACM Trans. Program. Lang. Syst., vol. 32, no. 5,2010

[36] S. D. Brookes, "A Semantics for Concurrent Separation Logic," in Concurrency Theory, 15th Int. Conference (CONCUR'04), 2004, pp. $16-34$. 


\section{Technical Report YALEU/DCS/TR-1476}

\section{APPENDix}

\section{Further Preliminary Explanations}

Permissions: It is sometimes necessary to share information in the form of a predicate between the invariant and a local assertion. This can be achieved in CSL by the use of permissions [21].

The predicate $E \mapsto F$ expresses that the heap location denoted by $E$ contains the value that $F$ denotes. Another natural reading of the predicate in the context of separation logic is that $E \mapsto F$ grants the permissions of reading from and writing to the heap location denoted by $E$ (permission reading). Building upon this interpretation, read permissions state that a full read/write permission $E \mapsto F$ can be shared by two threads if the heap location denoted by $E$ will not be modified. A full permission and two read permissions can be interchanged using the following equivalence. ${ }^{3}$

$$
E \mapsto F \Leftrightarrow E \stackrel{r}{\mapsto} F * E \mapsto r
$$

The two read permissions can then be shared between two threads. To write into a location, a thread needs a full permission and to read a location it only needs a read permission.

$$
\begin{gathered}
{\left[x \mapsto{ }_{-}\right][x]:=E[x \mapsto E] \text { (WRIte) }} \\
{[E \stackrel{r}{\mapsto} F] x:=[E][E \stackrel{r}{\mapsto} F \wedge x=F] \text { (READ) }}
\end{gathered}
$$

The remaining rules of the concurrent separation logic can remain unchanged in the presence of permissions.

Auxiliary Variables: If the rules of (concurrent) separation logic are not sufficient to prove a property of a program then we sometimes have to use auxiliary variables [20]. These are variables that we add to the program to monitor but not influence the computation of the original program. Thus, if we prove a property about a program using auxiliary variables then this property also holds for the program without the auxiliary variables.

More formally, we say a set $A u x$ of variables is auxiliary for a program $P$ if the following holds. If $x:=E$ is an assignment in $P$ and $E$ contains a variable in $A u x$ then $x \in$ Aux. Additionally, auxiliary variables must not occur in loop or conditional tests.

\section{Formal Development AND Soundness}

In the following, we give the formalization and soundness proof of our quantitative concurrent separation logic for total correctness. The proof is inspired by Vafeiadis' soundness proof [26] of concurrent separation logic and Atkey's soundness proof of his (sequential) quantitative separation logic [19]. However, we not only prove memory safety but also termination.

First, we address the syntax and semantics of our language and logic in detail. See Figure 8 for the full operational semantics of our language and Figure 9 for the Hoare-style derivation rules of the logic. The semantics are standard with

\footnotetext{
${ }^{3} \mathrm{~A}$ read permission is equivalent to a fractional permission with the fraction 0.5 .
}

$$
\begin{aligned}
E::= & x|n| E+E|E-E| \ldots \\
B::= & E=E|E<E| \neg B|B \vee B| \ldots \\
C::= & \operatorname{skip}|x:=E| x:=[E]|[E]:=E| x:=\operatorname{alloc}(n) \\
& \mid \text { dispose }(E)|C ; C| C|C| \text { if } B \text { then } C \text { else } C \\
& \mid \text { while } B \text { do } C \mid \text { atomic } C \mid\{C\}
\end{aligned}
$$

Fig. 6. A basic while language with concurrency and dynamic allocation.

the exception of the WHILE-LOOP, WHILE-SKIP, and WHILEABORT rules, which deal with safe and unsafe loops in a program. Similarly, the derivation rules include an extended WHILE rule that provides a logical specification that ensures that while loops are terminating.

Language: We use a basic while language with concurrency as commonly used in the context of concurrent separation logic [36], [14], [26]. As defined in Figure 6, it is built from integer expressions $E$, boolean expressions $B$, and commands $C$. As in Parkinson et al. [18], we assume a global shared heap region. An extension to conditional critical regions [14] is possible but omitted in favor of clarity. We assume that each built-in function terminates. For simplicity, we do not include procedure calls in the language. This is an orthogonal issue that is dealt with elsewhere [27].

Semantics: Formulas and programs are interpreted with respect to a program state using a small-step operational semantics. Since the logic includes a consumable resource predicate, a program state consists not only of a heap and a stack but also of a natural number $t$ which represents the number of consumable resources that are currently available to the program. To execute the body of a while loop there has to be at least 1 resource available, that is $t>0$. After the execution of the loop body, there are $t-1$ resources left.

Let $S t a c k=\operatorname{Var} \rightarrow$ Val be the set of stacks and Heap $=$ $L o c \rightarrow{ }_{\text {fin }} V a l$ be the set of heaps. Then, the set of program states is State $=$ Heap $\times$ Stack $\times \mathbb{N}$. The last component describes the number of available tokens.

The rules of the semantics are defined in Figure 8. They define an evaluation judgment of the forms

$$
C, \sigma \rightarrow C^{\prime}, \sigma^{\prime} \quad \text { or } \quad C, \sigma \rightarrow \perp
$$

where $C$ and $C^{\prime}$ are commands and $\sigma, \sigma^{\prime} \in$ State. Intuitively, this judgment states the following. If we execute the command $C$ in the state $\sigma$ then the next computational step results in an error $(C, \sigma \rightarrow \perp)$, or it transforms the program state to $\sigma^{\prime}$ and execution continues with command $C^{\prime}$. A deviation from the standard rules is in the semantics for a while loop. They ensure that a token is consumed if the body of the loop is executed. If the loop condition is satisfied and no token is available $(t=0)$ in the current state, then the result is an error.

An interesting feature of our semantics is that it does not admit infinite chains of execution steps. We prove this by defining a well-founded order $\prec$ on program states and commands. To this end, we first define the size $|C|$ of a command $C$ as follows. 


\section{Technical Report YALEU/DCS/TR-1476}

Definition 1 (Size of Commands). Let $C$ be a command. $|C|$ is inductively defined as follows.

$$
\begin{aligned}
\mid \text { skip } \mid & =0 \\
\left|C_{1} ; C_{2}\right| & =\left|C_{1}\right|+\left|C_{2}\right|+1 \\
\left|C_{1}\right|\left|C_{2}\right| & =\left|C_{1}\right|+\left|C_{2}\right|+1 \\
\mid \text { if } B \text { then } C_{1} \text { else } C_{2} \mid & =\max \left(\left|C_{1}\right|,\left|C_{2}\right|\right)+1 \\
\mid \text { while } B \text { do } C \mid & =|C|+1 \\
\mid \text { atomic } C \mid & =|C|+1 \\
|\{C\}| & =|C| \\
|C| & =1 \quad \text { otherwise }
\end{aligned}
$$

Definition 2 (Well-Founded Order). Let $\sigma=(H, V, t), \sigma^{\prime}=$ $\left(H^{\prime}, V^{\prime}, t^{\prime}\right)$ be program states and let $C, C^{\prime}$ be commands. We define $\left(C^{\prime}, \sigma^{\prime}\right) \prec(C, \sigma)$ iff $t^{\prime}<t$ or $\left(t^{\prime}=t\right.$ and $\left.\left|C^{\prime}\right|<|C|\right)$.

Proposition 1. The relation $\prec$ is a well-founded order on program states.

Lemma 1. If $C, \sigma \rightarrow C^{\prime}, \sigma^{\prime}$ then $\left(C^{\prime}, \sigma^{\prime}\right) \prec(C, \sigma)$.

Proof. By inspection of the operational semantics rules.

As a consequence of Lemma 1 and the well-foundedness of $<$ on the natural numbers, there are no infinite chains of the form $C_{1}, \sigma_{1} \rightarrow C_{2}, \sigma_{2} \rightarrow \cdots$.

Theorem 2. There exist no infinite chains of the form $C_{1}, \sigma_{1} \rightarrow$ $C_{2}, \sigma_{2} \rightarrow \cdots$.

Definition 3. For a program state $\sigma$ and a command $C$ we write $C, \sigma \Downarrow \sigma^{\prime}$ if $C, \sigma \rightarrow^{*}$ skip, $\sigma^{\prime}$. Similarly, we write $C, \sigma \Downarrow$ $\perp$ if $C, \sigma \rightarrow^{*} \perp$.

An inspection of the rules of the operational semantics shows that each terminal state has the form skip, $\sigma$.

Definition 4 (Termination). We say that a program $C$ terminates from an initial state $\sigma$ if not $C, \sigma \Downarrow \perp$.

Because our semantics do not allow infinite evaluation chains, we relate this definition to a usual small-step semantics without tokens. Since this relation is not important for the formal development we keep the discussion short. This semantic judgement is of the form $C, \tau \Rightarrow C^{\prime}, \tau^{\prime}$ or $C, \tau \Rightarrow \perp$, where $C, C^{\prime}$ are commands and $\tau, \tau^{\prime} \in$ Heap $\times$ Stack. The rules of the semantics are identical to the rules of our quantitative semantics with the token component removed. The only exceptions are the rules for while loops which are replaced by the following rules.

$\frac{\llbracket B \rrbracket(V)}{\{\text { while } B \text { do } C\},(H, V) \Rightarrow\{C ; \text { while } B \text { do } C\},(H, V)}$ (W-Loop)

$$
\frac{\neg \llbracket B \rrbracket(V)}{\{\text { while } B \text { do } C\}, \tau \Rightarrow \text { skip, } \tau} \text { (W-SKIP) }
$$

Theorem 3. Let $C$ be a command and let $\sigma=(H, V, t)$ by a state. If not $C, \sigma \Downarrow \perp$ then there is no infinite chain of the form $C,(H, V) \Rightarrow C_{1}, \tau_{1} \Rightarrow C_{2}, \tau_{2} \Rightarrow \cdots$ and not $C,(H, V) \Rightarrow^{*} \perp$.

$$
\begin{aligned}
\sigma \models \diamond \Leftrightarrow & t>0 \wedge \operatorname{dom}(H)=\emptyset \\
\sigma \models P * Q \Leftrightarrow & \exists H_{1}, H_{2}, t_{1}, t_{2} . H=H_{1} \oplus H_{2} \wedge \\
& t=t_{1}+t_{2} \wedge\left(H_{1}, V, t_{1}\right) \models P \wedge \\
& \left(H_{2}, V, t_{2}\right) \models Q \\
\sigma \models P * Q \Leftrightarrow & \forall H^{\prime}, t^{\prime} . \text { if } H \oplus H^{\prime} \text { defined } \wedge\left(H^{\prime}, V, t^{\prime}\right) \models P \\
& \text { then }\left(H \oplus H^{\prime}, V, t+t^{\prime}\right) \models Q \\
\sigma \models E \mapsto F \Leftrightarrow & \operatorname{dom}(H)=\llbracket E \rrbracket(V) \\
& \wedge H(\llbracket E \rrbracket(V))=(\llbracket F \rrbracket(V), \top) \\
\sigma \models E \mapsto \mapsto^{r} F \Leftrightarrow & \operatorname{dom}(H)=\llbracket E \rrbracket(V) \\
& \wedge H(\llbracket E \rrbracket(V))=(\llbracket F \rrbracket(V), r)
\end{aligned}
$$

Fig. 7. A sample of the semantics of assertions over a state $\sigma=(H, V, t)$. The semantics of the other connectives and predicates are standard.

To prove the theorem, we first prove for every $t \in \mathbb{N}$ and every program state $\tau=(H, V)$ that if $C, \tau \Rightarrow C^{\prime},\left(H^{\prime}, V^{\prime}\right)$ then either $C,(H, V, t) \rightarrow C^{\prime},\left(H^{\prime}, V^{\prime}, t^{\prime}\right)$ for some $t^{\prime}$ or $C,(H, V, t) \rightarrow \perp$. This follows immediately by an inspection of the rules. The only interesting case is the treatment of while loops for which the property is easily verified.

Given this, we see that the notion of termination $C, \sigma \Downarrow \sigma^{\prime}$ corresponds exactly to the standard notion of termination under a semantics without a resource component.

Concurrent Separation Logic with Quantitative Reasoning: Following the presentation of Atkey [19], we define the predicates of quantitative separation logic as follows. Since we only deal with one resource at a time we write $\diamond$ instead of Atkey's $R$.

$$
\begin{aligned}
P::= & B|P \vee P| P \wedge P|\neg P| P \Rightarrow P|\forall x . P| \exists x . P \\
& |\diamond| e m p|E \mapsto E| E \mapsto E|P * P| P * Q \mid \underset{i \in I}{\circledast} P
\end{aligned}
$$

Following previous work [21], [26], we model assertions in the logic with permission heaps. Heap locations are instrumented with a permission in $\{r, \top\}$ where $r$ is read-only and $T$ is full permission. Permission heaps can be added using the $\oplus$ operator, which adds permissions where they overlap (and are both $r$ ), and takes the disjoint union elsewhere. The operational semantics is independent of the permissions. So we define it for heaps without permissions, which can be derived from permission heaps by deleting the permission component. Figure 7 contains the semantics of the most interesting connectives and predicates.

The rules of the program logic are given in Figure 9

Soundness: In keeping with the presentation given in [26], we define satisfaction of Hoare triples according to the inductively defined predicate $\operatorname{safe}_{n}(C, \sigma, I, Q)$ which states that command $C$ will execute safely for up to $n$ steps starting in state $\sigma$ under resource invariant $I$ and if it terminates, the resulting state will satisfy $Q$. 


$$
\begin{aligned}
& \frac{\ell=\llbracket E \rrbracket(V) \quad \ell \in \operatorname{dom}(H)}{\{x:=E\},(H, V, t) \rightarrow \operatorname{skip},\left(H,\left.V\right|_{x=\llbracket E \rrbracket(V)}, t\right)}\left(\text { Assign) } \quad \frac{\ell=[E]\},(H, V, t) \rightarrow \operatorname{skip},\left(H,\left.V\right|_{x=H(\ell)}, t\right)}{\text { (LOOKUP) }}\right. \\
& \frac{\llbracket E \rrbracket(V) \notin \operatorname{dom}(H)}{\{x:=[E]\},(H, V, t) \rightarrow \perp}\left(\text { LOOKUP-ABORT) } \quad \frac{\ell=\llbracket E \rrbracket(V) \quad \ell \in \operatorname{dom}(H)}{\{[E]:=F\},(H, V, t) \rightarrow \operatorname{skip},\left(\left.H\right|_{\ell=\llbracket F \rrbracket(V)}, V, t\right)}\right. \text { (MUTATE) } \\
& \frac{\llbracket E \rrbracket(V) \notin \operatorname{dom}(H)}{\{[E]:=F\},(H, V, t) \rightarrow \perp}\left(\text { MUtATE-ABORT) } \quad \frac{\forall i \in\{0, \ldots, n-1\} \cdot \ell+i \notin \operatorname{dom}(H)}{\{x:=\operatorname{alloc}(n)\},(H, V, t) \rightarrow \operatorname{skip},\left(\left.H\right|_{\ell+0, \ldots, \ell+n-1=0},\left.V\right|_{x=\ell}, t\right)}(\text { ALLOC })\right. \\
& \frac{\ell=\llbracket E \rrbracket(V) \quad \ell \in \operatorname{dom}(H)}{\operatorname{dispose}(E),(H, V, t) \rightarrow \operatorname{skip},(H \backslash \ell, V, t)} \text { (DISPOSE) } \quad \frac{\llbracket E \rrbracket(V) \notin \operatorname{dom}(H)}{\operatorname{dispose}(E),(H, V, t) \rightarrow \perp} \text { (DISPOSE-ABORT) } \\
& \frac{C_{1}, \sigma \rightarrow C_{1}^{\prime}, \sigma^{\prime}}{\left\{C_{1} ; C_{2}\right\}, \sigma \rightarrow\left\{C_{1}^{\prime} ; C_{2}\right\}, \sigma^{\prime}}(\mathrm{SEQ} 1) \quad \frac{C_{1}, \sigma \rightarrow \perp}{\left\{\text { skip; } C_{2}\right\}, \sigma \rightarrow C_{2}, \sigma}(\mathrm{SEQ} 2) \quad(\mathrm{SEQ}-\mathrm{ABORT}) \\
& \frac{C_{1}, \sigma \rightarrow C_{1}^{\prime}, \sigma^{\prime}}{\left\{C_{1} \| C_{2}\right\}, \sigma \rightarrow\left\{C_{1}^{\prime} \| C_{2}\right\}, \sigma^{\prime}}(\text { PAR } 1) \quad \frac{C_{2}, \sigma \rightarrow C_{2}^{\prime}, \sigma^{\prime}}{\left\{C_{1} \| C_{2}\right\}, \sigma \rightarrow\left\{C_{1} \| C_{2}^{\prime}\right\}, \sigma^{\prime}}\left(\text { PAR2) } \quad \frac{\{\text { skip } \| \text { skip }\}, \sigma \rightarrow \text { skip }, \sigma}{\text { (PAR3) }}\right. \\
& \frac{C_{1}, \sigma \rightarrow \perp}{\left\{C_{1} \| C_{2}\right\}, \sigma \rightarrow \perp}(\text { PAR-Abort } 1) \quad \frac{C_{2}, \sigma \rightarrow \perp}{\left\{C_{1} \| C_{2}\right\}, \sigma \rightarrow \perp}(\text { PAR-Abort } 2) \quad \frac{\llbracket B \rrbracket(V)}{\left\{\text { if } B \text { then } C_{t} \text { else } C_{f}\right\},(H, V, t) \rightarrow C_{t},(H, V, t)}(\mathrm{IF}-\mathrm{TrUE})
\end{aligned}
$$

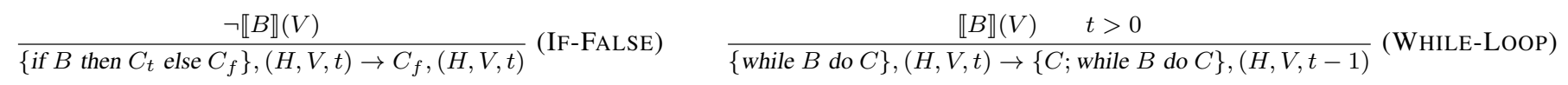

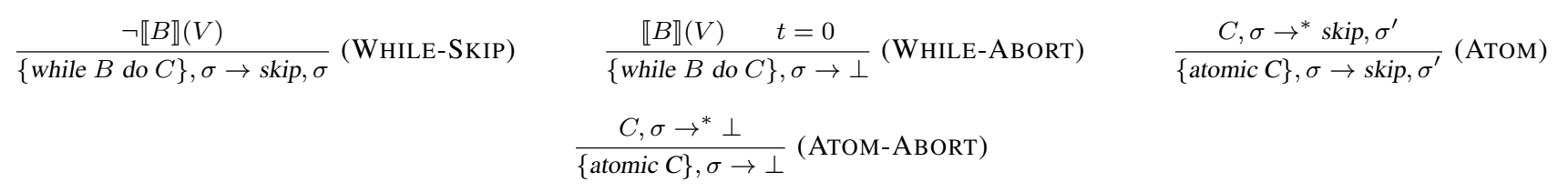

$$
\begin{aligned}
& \frac{x \notin \mathrm{fv}(I)}{I \vdash[P] \operatorname{skip}[P]}(\mathrm{SKIP}) \quad \frac{x \notin \mathrm{fv}(I, E, F)}{I \vdash[P[E / x]] x:=E[P]}(\mathrm{ASSIGN}) \quad \frac{r}{I \vdash[E \mapsto F] x:=[E][E \mapsto F \wedge x=F]}(\mathrm{LOOKUP}) \\
& \frac{x \notin \mathrm{fv}(I)}{I \vdash\left[E \mapsto \mapsto_{-}\right][E]:=F[E \mapsto F]} \text { (MUtATE) } \quad \frac{x \wedge \ldots \wedge x+n-1 \mapsto 0]}{I \vdash[e m p] x:=\operatorname{alloc}(n)[x+0 \mapsto 0 \wedge \ldots \text { (ALOC) }} \\
& \frac{I \vdash\left[E \mapsto{ }_{-}\right] \operatorname{dispose}(E)[e m p]}{I \vdash[P] C_{1}[Q] \quad I \vdash[Q] C_{2}[R]}(\mathrm{DISPOSE}) \quad \frac{I \vdash[P] C_{1} ; C_{2}[R]}{(\mathrm{SEQ})}
\end{aligned}
$$

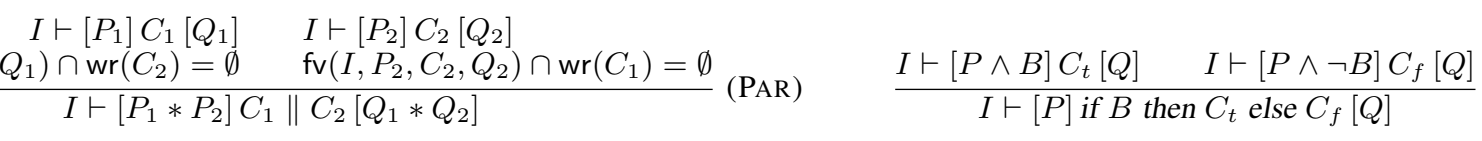

$$
\begin{aligned}
& \frac{P \wedge B \Longrightarrow P^{\prime} * \diamond \quad I \vdash\left[P^{\prime}\right] C[P]}{I \vdash[P] \text { while } B \operatorname{do} C[P \wedge \neg B]} \text { (WhILe) } \quad \frac{e m p \vdash[P * I] C[Q * I]}{I \vdash[P] \text { atomic } C[Q]} \text { (ATOM) } \quad \frac{I * J \vdash[P] C[Q]}{I \vdash[P * J] C[Q * J]} \text { (SHARE) } \\
& \frac{I \vdash[P] C[Q] \quad \mathrm{fv}(R) \cap \operatorname{wr}(C)=\emptyset}{I \vdash[P * R] C[Q * R]} \text { (Frame) } \quad \frac{I \vdash[P] C[Q] \quad P^{\prime} \Rightarrow P \quad Q \Rightarrow Q^{\prime}}{I \vdash\left[P^{\prime}\right] C\left[Q^{\prime}\right]} \text { (ConseQuence) } \\
& \frac{I \vdash\left[P_{1}\right] C[Q] \quad I \vdash\left[P_{2}\right] C[Q]}{I \vdash\left[P_{1} \vee P_{2}\right] C[Q]} \text { (Disjunction) } \quad \frac{I \vdash[P] C[Q] \quad x \notin \mathrm{fv}(C)}{I \vdash[\exists x . P] C[\exists x . Q]} \text { (Existential) } \\
& \frac{I \vdash[P] C\left[Q_{1}\right] \quad I \vdash[P] C\left[Q_{2}\right] \quad I \text { precise }}{I \vdash[P] C\left[Q_{1} \wedge Q_{2}\right]} \text { (CONJUNCTION) }
\end{aligned}
$$

Fig. 9. Derivation rules. $\mathrm{fv}$ gives the set of free variables in a command or predicate. wr gives the set of variables which are modified by a command. 
Definition 5 (Safety). For any state $\sigma=(H, V, t)$, command $C$, and predicates $I, Q$ :

- $\operatorname{safe}_{0}(C, \sigma, I, Q)$ holds.

- $\operatorname{safe}_{n+1}(C, \sigma, I, Q)$ holds when all of the following are true:

1) If $C=$ skip then $\sigma=Q$.

2) For all $t_{I} \in \mathbb{N}$ and all $H_{I}, H_{F} \in$ Heap such that $\left(H_{I}, V, t_{I}\right) \models I$ and $H \oplus H_{I} \oplus H_{F}$ is defined, $C,\left(H \oplus H_{I} \oplus H_{F}, V, t+t_{I}\right) \nrightarrow \rightarrow \perp$.

3) For all $t_{I}, t^{\prime} \in \mathbb{N}, H_{I}, H_{F}, H^{\prime} \in$ Heap, and $V^{\prime} \in$ Stack such that $\left(H_{I}, V, t_{I}\right) \models I$ and $H \oplus H_{I} \oplus$ $H_{F}$ is defined, if $C,\left(H \oplus H_{I} \oplus H_{F}, V, t+t_{I}\right) \rightarrow$ $C^{\prime},\left(H^{\prime}, V^{\prime}, t^{\prime}\right)$, then there exist $H^{\prime \prime}, H_{I}^{\prime}$ and $t^{\prime \prime}$ such that $H^{\prime}=H^{\prime \prime} \oplus H_{I}^{\prime} \oplus H_{F}, t^{\prime \prime} \leq t^{\prime},\left(H_{I}^{\prime}, V^{\prime}, t^{\prime \prime}\right) \models I$ and $\operatorname{safe}_{n}\left(C^{\prime},\left(H^{\prime \prime}, V^{\prime}, t^{\prime}-t^{\prime \prime}\right), I, Q\right)$.

When $n>0$, the first condition specifies that if the execution is in a terminal state, then that state satisfies the postcondition $Q$. The second condition states that the execution will not go wrong. The third condition ensures that each step preserves the resource invariant $I$, and that after executing one step, the resulting program is safe for another $n-1$ steps. In the second and third conditions, $H_{I}$ and $t_{I}$ represent the resources required to satisfy the global invariant $I . H_{F}$ represents additional heap cells which may be needed by other parts of the program. Note that we do not include a frame $t_{F}$ of consumable resources. Since predicate satisfaction is monotonic with respect to consumable resources, we do not need to distinguish between consumable resources in the shared region $\left(t_{I}\right)$ and those in the frame. Also, since the operational semantics only work on concrete heaps, in condition (3) $H_{F}$ will necessarily contain any heap locations that an executing thread has read permission to. By using the same $H_{F}$ before and after an execution step we thus ensure that a thread cannot modify a heap location unless it has full permission at that location.

Given this, we say that a Hoare triple $[P] C[Q]$ is satisfiable under an invariant $I$ if and only if for all $n \in \mathbb{N}$ and all states $\sigma \models P$, $\operatorname{safe}_{n}(C, \sigma, I, Q)$ holds. For a discussion of the motivations behind this particular characterisation of satisfaction, see [26].

Before we present the proof of soundness of the logic, we need to consider two aspects of the logic and how they interact with consumable resources: Permission Heaps [21] and Precise Assertions [14].

Permission Heaps: Let Perm $=\{r, \top\}$ be a permissions set, with $r$ indicating read-only permission and $T$ indicating full permission. Then, let PHeap $=L o c \rightarrow_{f i n}$ Val $\times$ Perm be the set of permission heaps. A permission heap $H \in$ PHeap is a finite mapping from locations to pairs of values and permissions. Perm is equipped with a commutative partial operator $\oplus$ defined as $r \oplus r=\top$, and undefined otherwise.

We extend the permission operator $\oplus$ to value-permission pairs as follows:

$\left(v_{1}, p_{1}\right) \oplus\left(v_{2}, p_{2}\right)= \begin{cases}\left(v_{1}, \top\right) & \text { if } v_{1}=v_{2} \text { and } p_{1}=p_{2}=r \\ \text { undefined } & \text { otherwise }\end{cases}$
We further extend $\oplus$ to permission heaps $H_{1}$ and $H_{2}$ that agree on the values at overlapping locations:

$\left(H_{1} \oplus H_{2}\right)(\ell)= \begin{cases}H_{1}(\ell) \oplus H_{2}(\ell) & \text { if } \ell \in \operatorname{dom}\left(H_{1}\right) \cap \operatorname{dom}\left(H_{2}\right) \\ H_{1}(\ell) & \text { if } \ell \in \operatorname{dom}\left(H_{1}\right) \backslash \operatorname{dom}\left(H_{2}\right) \\ H_{2}(\ell) & \text { otherwise }\end{cases}$

Given this, we model assertions in the logic with permission heaps (see Figure 7). As in Vafeiadis [26], assertions are modeled with permission heaps but the operational semantics act on concrete heaps. To reconcile this, we consider regular heaps as a subset of permission heaps where the permission is always $T$

$$
\text { Heap }=L o c \rightarrow_{\text {fin }} \operatorname{Val} \times\{\top\}
$$

Then, for any permission heap $H$ there exists a complementary permission heap $H^{\prime}$ for which $H \oplus H^{\prime}$ is a concrete heap. Specifically, $H^{\prime}$ must contain the sub-heap of $H$ that includes all the locations at which $H$ has read permission. Define $\operatorname{read}(H)$ to be such a sub-heap:

$$
\operatorname{read}(H) \triangleq\{(v, p) \mid(v, p) \in H \wedge p=r\}
$$

Then,

$$
\forall H \in \text { PHeap. } H \oplus \operatorname{read}(H) \in \text { Heap }
$$

Now consider Definition 5 of $\operatorname{safe}_{n}(C, \sigma, I, Q)$. In the definition, every time the small-step judgement $\rightarrow$ is invoked, the heap is $H \oplus H_{I} \oplus H_{F}$, which includes the universally quantified $H_{F}$. Thus, $H_{F}$ will always include $\operatorname{read}(H) \oplus \operatorname{read}\left(H_{I}\right)$. This means that $H \oplus H_{I} \oplus H_{F}$ is a concrete heap, so the definition makes sense. Furthermore, since $H_{F}$ is not modified by the step in condition (3), $C$ cannot modify locations in the heap to which $H$ has only read access.

Precise Assertions: As shown in [14], [26], in order for the logic to be sound, we require that the global resource invariant be precise in the CONJUNCTION rule. We define precise assertions [14], [26] as follows. An assertion $P$ is precise when it is satisfied by exactly one sub-heap of any heap.

Definition 6 (Precise Assertions). Let $V \in$ Stack, $t \in \mathbb{N}$ and let $P$ be an assertion. $P$ is precise if and only if for all $H_{1}, H_{2}, H_{1}^{\prime}, H_{2}^{\prime} \in P$ Peap such that $H_{1} \oplus H_{2}$ is defined and $H_{1} \oplus H_{2}=H_{1}^{\prime} \oplus H_{2}^{\prime}$, if $\left(H_{1}, V, t\right) \models P$ and $\left(H_{1}^{\prime}, V, t\right) \models P$, then $H_{1}=H_{1}^{\prime}$.

This definition does not consider our consumable resources. Since the assertion $\diamond^{k}$ is satisfiable by any set of at least $k$ resources, it is impossible for a predicate to specify an exact set of resources. Regardless, since the tokens are affine entities as we see in the following proof, the soundness of the logic is unaffected.

Before we prove the soundness of the rules, we have to prove two additional lemmas that are needed in the case of the rule WHILE.

Lemma 2. Let $\sigma=(H, V, t)$ be a program state, $I, Q, R$ predicates, $C_{1} ; C_{2}$ a command, and $n$ a natural number. If safe $_{n}\left(C_{1}, \sigma, I, Q\right)$ and for all $m \leq n$ and $\sigma^{\prime}$ with $\sigma^{\prime} \models Q$, safe $_{m}\left(C_{2}, \sigma^{\prime}, I, R\right)$ then safe $_{n}\left(C_{1} ; C_{2}, \sigma, I, R\right)$. 
The proof of Lemma 2 is identical to the proof of the same lemma in (the formalized proof of) [26].

Lemma 3. Let $\sigma=(H, V, t)$ be a program state, while $B$ do $C$ a command, and $I, P, P^{\prime}$ predicates such that $P \wedge B \Longrightarrow P^{\prime} * \diamond$. If $\left[P^{\prime}\right] C[P]$ is satisfiable under $I$ and $\sigma \models P$ then for all $n$, safe ${ }_{n}$ (while $B$ do $C, \sigma, I, P \wedge \neg B$ ).

Proof. We prove the lemma by induction on $n$. The base case for $n=0$ follows directly from the definition of safe $\mathrm{s}_{0}$.

Assume now that safe ${ }_{n}$ (while $B$ do $C, \sigma, I, P \wedge \neg B$ ) holds. To show safe ${ }_{n+1}$ (while $B$ do $C, \sigma, I, P \wedge \neg B$ ), we show that all three conditions in Definition 5 are satisfied:

1) We have while $B$ do $C \neq$ skip, so this condition holds vacuously.

2) The only rule in the operational semantics which can derive $\{$ while $B$ do $C\}, \sigma \rightarrow \perp$ is While-Abort. The premises of the rule are $\llbracket B \rrbracket(V)$ and $t=0$. We show that from $\llbracket B \rrbracket(V)$ it follows that $t>0$. Therefore WHILEABORT does not apply. Assume $\llbracket B \rrbracket(V)$. Since $\sigma \models P$ we have then $\sigma \mid=P \wedge B$, and thus it follows from the premises that $\sigma \models P^{\prime} * \diamond$. From the semantics of $\diamond$ we derive $t \geq 1$. This confirms condition (2).

3) Let $t_{I}, t^{\prime} \in \mathbb{N}, H_{I}, H_{F} \in$ PHeap, $H^{\prime} \in$ Heap and $V^{\prime} \in$ Stack such that $\left(H_{I}, V, t_{I}\right) \models I$ and $H \oplus H_{I} \oplus$ $H_{F}$ is defined, and $C,\left(H \oplus H_{I} \oplus H_{F}, V, t+t_{I}\right) \rightarrow$ $C^{\prime},\left(H^{\prime}, V^{\prime}, t^{\prime}\right)$. Then the rules WhILE-Loop or WHILESKIP have been applied. Since non of these rules modifies the heap (nor stack), $H^{\prime}=H \oplus H_{I} \oplus H_{F}$, so let $H^{\prime \prime}=H$ and $H_{I}^{\prime}=H_{I}$.

In the case of the rule WHILE-Loop, we have $C^{\prime}=$ $C$; while $B$ do $C$ and $t^{\prime}=t+t_{I}-1$. Let now $t^{\prime \prime}=$ $t_{I}$. Then $t \geq 1$ (premise of WHILE-Loop) and we have $t-1 \geq 0$, so $t^{\prime \prime} \leq t^{\prime}$. It follows by construction, $\left(H_{I}^{\prime}, V^{\prime}, t^{\prime \prime}\right)=\left(H_{I}, V, t_{I}\right)$, which satisfies $I$. Moreover $\left(H^{\prime \prime}, V^{\prime}, t^{\prime}-t^{\prime \prime}\right)=P^{\prime}$. Since $\left[P^{\prime}\right] C[P]$ is satisfiable under $I$, we have $\operatorname{safe}_{n}\left(C,\left(H^{\prime \prime}, V^{\prime}, t^{\prime}-t^{\prime \prime}\right), I, P\right)$. By induction we have safe s $_{m}$ while $B$ do $C, \sigma^{\prime}, I, P \wedge \neg B$ ) for all $m \leq n$ and all $\sigma^{\prime}$ with $\sigma^{\prime} \models P$. Therefore we derive $\operatorname{safe}_{n}\left(C^{\prime},\left(H^{\prime \prime}, V^{\prime}, t^{\prime}-t^{\prime \prime}\right), I, P\right)$ with Lemma 2. In the case of the rule WHILE-SKIP, we have $C^{\prime}=$ skip and $t^{\prime}=t+t_{I}$. Let again $t^{\prime \prime}=t_{I}$. Then $t^{\prime \prime} \leq t^{\prime}$ and it follows that $\left(H_{I}^{\prime}, V^{\prime}, t^{\prime \prime}\right)=\left(H_{I}, V, t_{I}\right)$ satisfies $I$. Furthermore, $\left(H^{\prime \prime}, V^{\prime}, t^{\prime}-t^{\prime \prime}\right) \models P$ and from the premise of the WHILE-SKIP we obtain $\left(H^{\prime \prime}, V^{\prime}, t^{\prime}-t^{\prime \prime}\right) \models P \wedge \neg B$. Thus safe ${ }_{n}$ (skip, $\left.\left(H^{\prime \prime}, V^{\prime}, t^{\prime}-t^{\prime \prime}\right), I, P \wedge \neg B\right)$. (Condition (1) follows from the aforesaid and Conditions (2) and (3) by inspection of the evaluation rules.)

Theorem 4 (Partial Correctness). For any propositions $I, P, Q$ and any command $C$, if $I \vdash[P] C[Q]$, then $[P] C[Q]$ is satisfiable under $I$.

Proof. The proof is by structural induction over the derivation rules given in Figure 9. Since the only command which accesses the resource component of program state is the while loop, the proof of every rule is essentially the same as in Vafeiadis [26] except for the rule WHILE. For all of the following, let $\sigma=$ $(H, V, t) \in$ State, $C$ be a command, and $I, P, Q$ be predicates.

While: Follows directly from Lemma 3.

Conjunction: To see that the definition of precise assertions is sufficient, we consider the CONJUNCTION rule. Let $I$ be a precise assertion, $Q_{1}, Q_{2}$ be any assertions, and let $C$ be a command. We show by induction that for any state $\sigma=(H, V, t)$ and any $n \in \mathbb{N}$, if $\operatorname{safe}_{n+1}\left(C, \sigma, I, Q_{1}\right)$ and safe $_{n+1}\left(C, \sigma, I, Q_{2}\right)$ then $\operatorname{safe}_{n+1}\left(C, \sigma, I, Q_{1} \wedge Q_{2}\right)$. Again, we confirm each condition:

1) if $C=$ skip, then $\sigma \models Q_{1}$ and $\sigma \models Q_{2}$. Thus, $\sigma \models$ $Q_{1} \wedge Q_{2}$.

2) Since this condition does not depend on the postcondition, it is already verified by the assumption safe $_{n+1}\left(C, \sigma, I, Q_{1}\right)$.

3) Let $t_{I}, t^{\prime} \in \mathbb{N}, H_{I}, H_{F} \in$ PHeap, $H^{\prime} \in$ Heap and $V^{\prime} \in$ Stack such that $\left(H_{I}, V, t_{I}\right) \models I$ and $H \oplus H_{I} \oplus H_{F}$ is defined, and assume that $C,\left(H \oplus H_{I} \oplus H_{F}, V, t+t_{I}\right) \rightarrow$ $C^{\prime},\left(H^{\prime}, V^{\prime}, t^{\prime}\right)$.

By our assumption, there exist $H^{\prime \prime 1}, H_{I}^{\prime 1}$ and $t^{\prime \prime 1}$ such that $H^{\prime}=H^{\prime \prime 1} \oplus H_{I}^{\prime 1} \oplus H_{F}, t^{\prime \prime 1} \leq t^{\prime},\left(H_{I}^{\prime 1}, V^{\prime}, t^{\prime \prime 1}\right) \models I$ and safe $_{n}\left(C^{\prime},\left(H^{\prime \prime 1}, V^{\prime}, t^{\prime}-t^{\prime \prime 1}\right), I, Q_{1}\right)$. Likewise for $H^{\prime \prime 2}, H_{I}^{\prime 2}, t^{\prime \prime 2}$ and $Q_{2}$.

This implies that $H^{\prime \prime 1} \oplus H_{I}^{\prime 1}=H^{\prime \prime 2} \oplus H_{I}^{\prime 2}$. Since $I$ is precise, we know that $H_{I}^{\prime 1}=H_{I}^{\prime 2}$, and thus $H^{\prime \prime 1}=H^{\prime \prime 2}$. Finally, let $t^{\prime \prime}=\min \left(t^{\prime \prime 1}, t^{\prime \prime 2}\right)$. Then, $t^{\prime}-t^{\prime \prime}$ will be at least as large as both $t^{\prime}-t^{\prime \prime 1}$ and $t^{\prime}-t^{\prime \prime 2}$ and will thus be sufficient to ensure that both $Q_{1}$ and $Q_{2}$ hold if the execution terminates. We conclude that safe $_{n}\left(C^{\prime},\left(H^{\prime \prime 1}, V^{\prime}, t^{\prime}-t^{\prime \prime 1}\right), I, Q_{1} \wedge Q_{2}\right)$.

The total correctness of the logic is a direct consequence of Theorem 4 and Theorem 2.

Theorem 5 (Total Correctness). Let I, P, Q be propositions, $C$ be a command, and $\sigma$ be a program state. If $\sigma \models P * I$ and $I \vdash[P] C[Q]$ then every evaluation of $C$ from the initial state $\sigma$ terminates in state $\sigma^{\prime}$ with $\sigma^{\prime} \models Q * I$.

\section{Memory SAfety of Treiber's Stack}

To additionally verify memory safety, we have to add some auxiliary state and extend our resource invariant. The verification is then similar to the proofs in related work on verification of safety properties [32], [33]. However, there are synergies between the lock-freedom and the memory safety proof.

See Figure 10 for the full implementation of Treiber's stack in our while language. The crucial point in the verification of memory safety are the assignments $x:=[t+1]$ and ret_val $:=[t]$ in the method pop. Our goal is to ensure, using the resource invariant, that these locations are owned by the shared region. At the evaluation of each assignment there are two possible cases: Either the memory location that is read is still part of the stack $S$ or it has been removed from the stack by another thread. To keep track of the memory locations that 

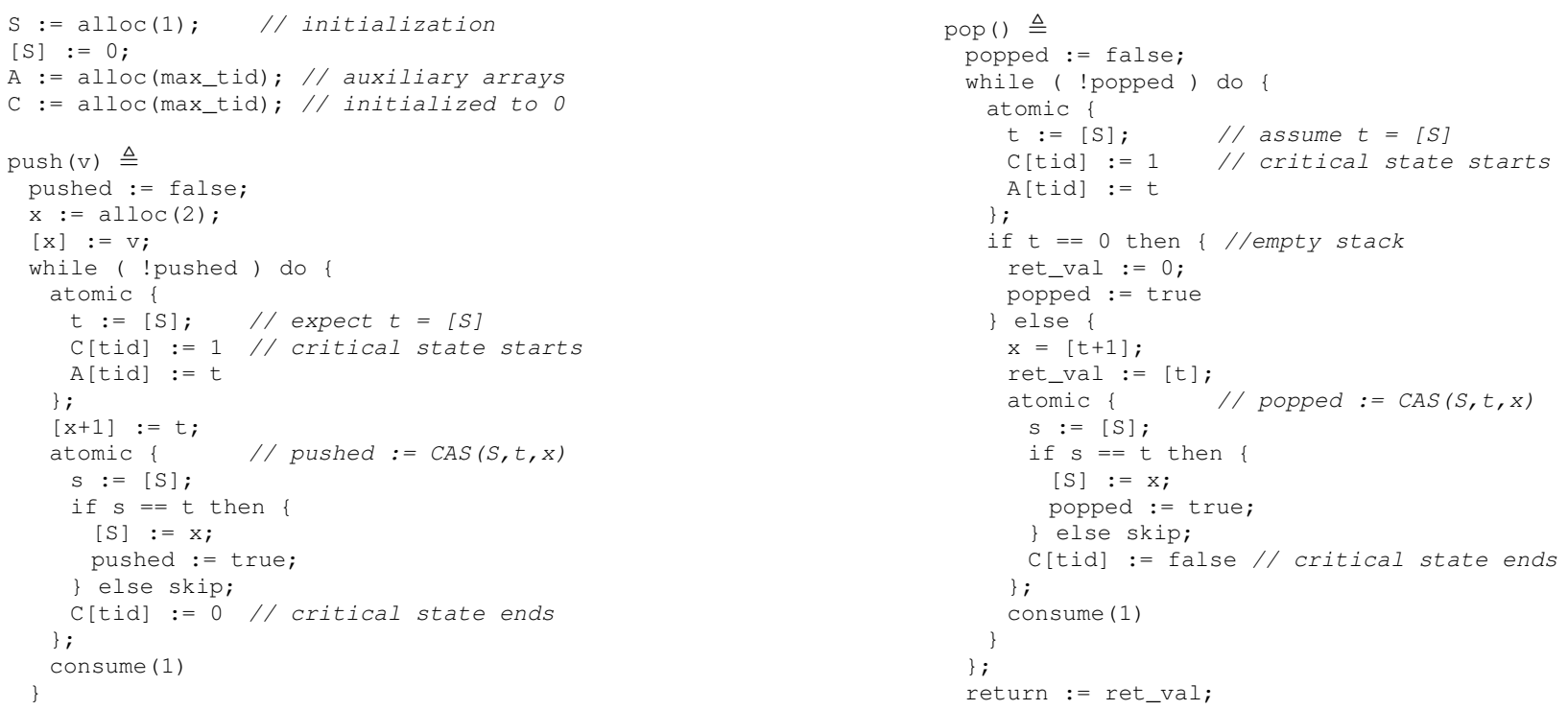

Fig. 10. A full implementation of Treiber's lock-free stack in our while language.

are pointed to by the stack, we introduce an inductive list predicate to describe the list pointed to by $S$. To keep track of the locations that have been removed from the stack we introduce an auxiliary variable that points to a second stack $G$ that contains all the locations that have been removed from $S$. To this end, we push a node onto $G$ after it is removed from $S$. That is, we replace the last atomic block in pop with the following code.

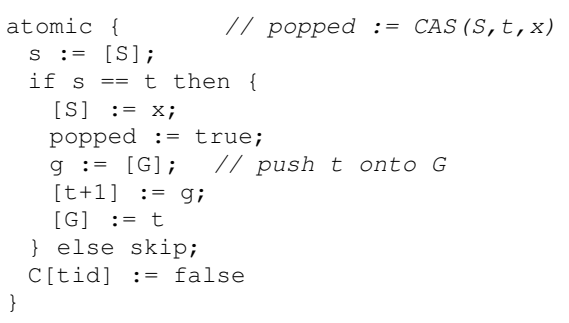

The invariant $I$ is then extended as follows where $n$ is again the total number of threads and $\alpha(i, u)$ is defined as before.

$$
\begin{aligned}
I^{\prime} \triangleq & \exists u . S \mapsto u * \underset{0 \leq i<n}{\circledast} \alpha(i, u) * G \mapsto v \\
& *\left(\exists u^{\prime}, v^{\prime} \operatorname{LSeg}\left(u, u^{\prime}\right) * \operatorname{LSeg}\left(v, v^{\prime}\right)\right) \wedge \\
& \bigwedge_{0 \leq i<n} \beta(i, u, v) \\
\beta(i, u, v) \triangleq & \exists a, c . C[i] \mapsto \mapsto^{r} c * A[i] \mapsto \mapsto_{\mapsto} a \\
& *(c=0 \vee \operatorname{LSeg}(u, a) \vee \operatorname{LSeg}(v, a))
\end{aligned}
$$

The inductive list predicate $L S e g$ is defined as usual [22] by

$$
\begin{aligned}
\operatorname{LSeg}(x, y) \Leftrightarrow & (x=y \wedge e m p) \vee \\
& (\exists v, z x \mapsto v * x+1 \mapsto z * \operatorname{LSeg}(z, y))
\end{aligned}
$$

The invariant ensures for each thread which is in the critical section that the local variable $t$ points to a location that is used by the lists pointed to by $S$ and $G$. Note that we can reuse the axillary arrays $A$ and $C$ in the formulas $\beta(i, u, v)$.

\section{VERIFICATION OF HENDLER ET AL'S ELIMINATION-BACKOFF STACK}

To improve the performance of Treiber's non-blocking stack in the presence of high contention, one can use an elimination backoff scheme [9]. The idea is based on the observation that a push operation followed by a pop results in a stack that is identical to the initial stack. So, if a stack operation fails because of the interference of another thread then the executing thread does not immediately retry the operation. Instead, it checks if there is another thread that is trying to perform a complementary operation. In this case, the two operations can be eliminated without accessing the stack at all: The two threads use a different shared-memory cell to transfer the stack element.

Our method can also be used to prove that Hendler et al's elimination-backoff stack [9] is lock-free. The main challenge in the proof is that the push and pop operations consist of two nested loops that are guarded by CAS operations. Assume again a system with $n$ threads. The inner loop can be just treated as in Treiber's stack using $n$ tokens in the precondition and 0 tokens in the postcondition. As a result, the number of tokens needed for an iteration of the outer loop is $n+1$. That means that a successful thread needs to transfer $(n-1) \cdot(n+1)=n^{2}-1$ tokens to the other threads to account for additional loop iterations in the other threads. Given this, we can verify the elimination-backoff stack using $n^{2}$ tokens in the precondition. Technically, we need an invariant of the form $I * J$, where $I$ is an invariant like in Treiber's stack (for the inner loop) and $J$ is like $I$ but with every token $\diamond$ replaced by $\diamond^{n}$.

To make this reasoning more concrete, Figure 11 shows the loop structures of the push operation of Hendler et al's stack 


\section{Technical Report YALEU/DCS/TR-1476}

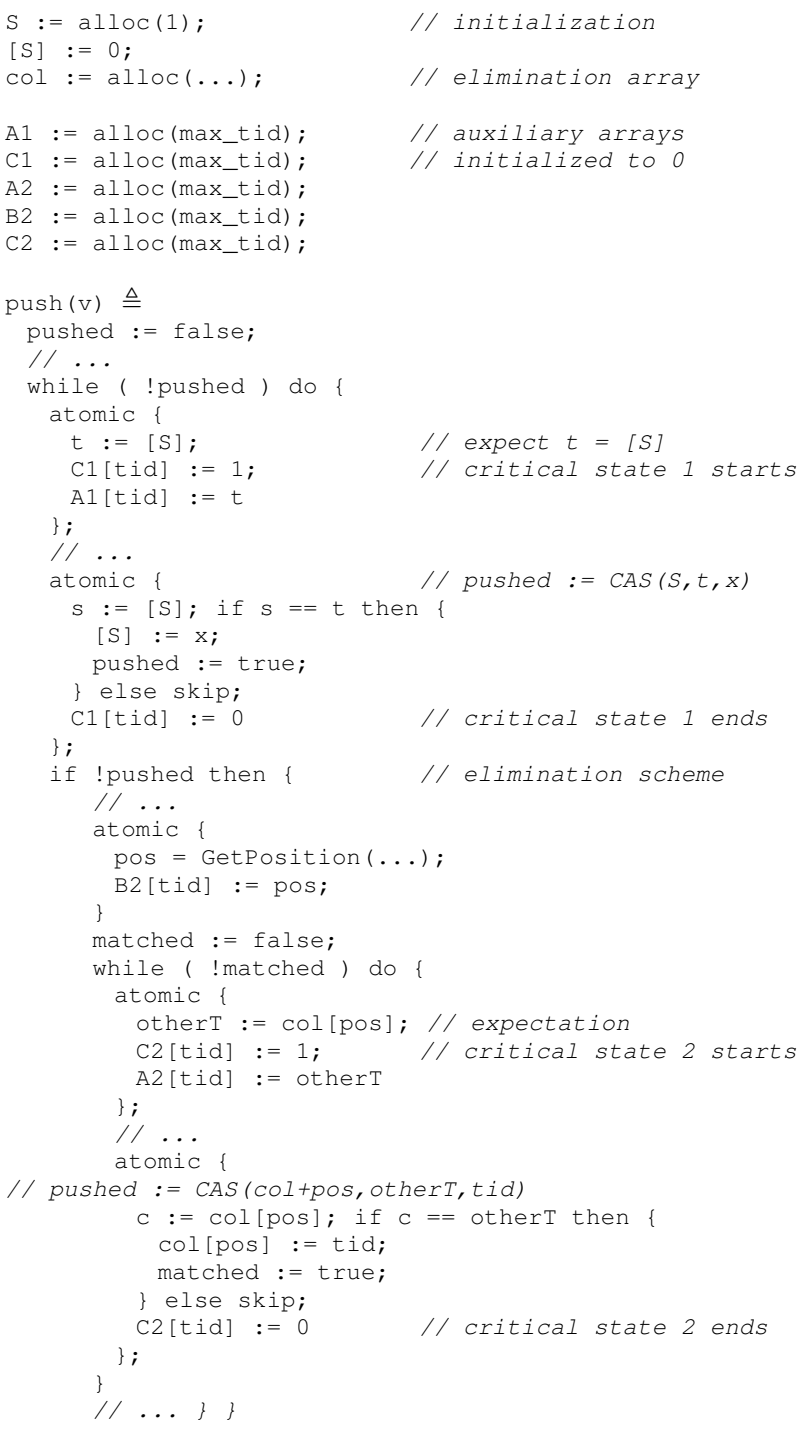

Fig. 11. The loop-structure of the push operation of Hendler et al's stack with elimination backoff scheme [9].

with elimination scheme in our while language. The auxiliary arrays $A 1$ and $C 1$ have the same purpose as in Treiber's stack: $C 1[t i d]$ indicates if thread tid is making an assumption on the value of the stack pointer $S$ and $A 1[t i d]$ contains the value of the local variable $t$. They will be used to formulate the part of the global invariant that is crucial to maintain the loop invariant of the outer while loop. The inner while loop has the same structure as the outer loop since it is also guarded by a CAS operation. However, the address on which the CAS is performed is not fixed. Thus we need three additional auxiliary arrays to formulate part of the global invariant that is needed for the inner loop: $C 2[t i d]$ indicates whether thread tid is making an assumption on the shared state that is stored in otherT. The array $B 2$ stores the memory address that is affected by this assumption and the array $A 2$ stores what the assumption is.
The global invariant $I$ can then be defined as follows.

$$
\begin{aligned}
& I \triangleq \exists u, v_{1}, \ldots, v_{n} . S \mapsto u * \underset{0 \leq i<n}{\circledast}(\delta(i, u) * \zeta(i)) \\
& *\left(\underset{0 \leq j<m}{\circledast} \operatorname{col}[j] \mapsto{ }_{-}{ }^{\circledast} \underset{0 \leq i<n}{\circledast} B 2[i] \stackrel{r}{\mapsto}{ }_{-} \wedge\right. \\
& \left.\bigwedge_{0 \leq i<n} \phi(i)\right) \\
& \delta(i, u) \triangleq \exists a, c . C 1[i] \stackrel{r}{\mapsto} c * A 1[i] \stackrel{r}{\mapsto} a *\left(c=0 \vee a=u \vee \diamond^{n}\right) \\
& \zeta(i) \triangleq \exists a, c . C 2[i] \stackrel{r}{\mapsto} c * A 2[i] \stackrel{r}{\mapsto} a *\left(c=0 \vee a=v_{i} \vee \diamond\right) \\
& \phi(i) \triangleq \exists b .0 \leq b<m * B 2[i] \stackrel{r}{\mapsto} b * \operatorname{col}[b] \mapsto v_{i}
\end{aligned}
$$

The formulas $\delta(i, u)$ are similar to the formulas $\alpha(i, u)$ in the invariant that we used to verify Treiber's stack. However, the single token $\diamond$ is replaced by $n$ tokens $\diamond^{n}$. The formulas $\zeta(i)$ are based on the same idea but are a bit more complicated since it is dynamically decided to which memory cell the assumption of thread $i$ applies (namely, $\operatorname{col}[b]$ contains the value that is stored in the thread-local variable other $T$ ). The formulas $\phi(i)$ form an invariant that relates the variables $v_{i}$ to the value stored in $\operatorname{col}[B 2[i]]$. The loop invariant of the outer loop is

$$
\begin{aligned}
& \left(\text { pushed } \vee \diamond^{n \cdot n}\right) * A 1[t i d] \stackrel{r}{\mapsto}{ }_{-} * C 1[t i d] \stackrel{r}{\mapsto}{ }_{-} \\
& * A 2[\text { tid }] \stackrel{r}{\mapsto} \text { - } * 2[\text { tid }] \stackrel{r}{\mapsto}{ }_{-} * C 2[\text { tid }] \stackrel{r}{\mapsto}{ }_{-}
\end{aligned}
$$

The loop invariant of the inner loop is

$$
\left(\text { matched } \vee \diamond^{n}\right) * A 2[\text { tid }] \stackrel{r}{\mapsto} \text { _ } * B 2[\text { tid }] \stackrel{r}{\mapsto} \text { _ } * C 2[\text { tid }] \stackrel{r}{\mapsto} \text {. }
$$

The proof is similar to the proof of Treiber's stack. 